\title{
RESTRICTION OF FOURIER TRANSFORMS TO CURVES AND RELATED OSCILLATORY INTEGRALS
}

\author{
JONG-GUK BAK DANIEL M. OBERLIN ANDREAS SEEGER
}

\begin{abstract}
We prove sharp endpoint results for the Fourier restriction operator associated to nondegenerate curves in $\mathbb{R}^{d}, d \geq 3$, and related estimates for oscillatory integral operators. Moreover, for some larger classes of curves in $\mathbb{R}^{d}$ we obtain sharp uniform $L^{p} \rightarrow L^{q}$ bounds with respect to affine arclength measure, thereby resolving a problem of Drury and Marshall.
\end{abstract}

\section{INTRODUCTION}

For a Schwartz function $f$ defined on $\mathbb{R}^{d}, d \geq 2$ consider the restriction of its Fourier transform to the curve $t \mapsto\left(t, t^{2}, \ldots, t^{d}\right)$ i.e.,

$$
\mathcal{R} f(t)=\widehat{f}\left(t, t^{2}, \ldots, t^{d}\right) .
$$

The problem of $L^{p}\left(\mathbb{R}^{d}\right) \rightarrow L^{q}(\mathbb{R})$ bounds is understood, namely $\mathcal{R}$ is bounded from $L^{p}\left(\mathbb{R}^{d}\right)$ to $L^{q}(\mathbb{R})$ if and only if

$$
1 \leq p<p_{d}:=\frac{d^{2}+d+2}{d^{2}+d} \quad \text { and } p^{\prime}=\frac{d(d+1)}{2} q ;
$$

likewise, if $I$ is a compact interval, then, for the same range of $p, \mathcal{R}$ is bounded from $L^{p}\left(\mathbb{R}^{d}\right)$ to $L^{q}(I)$ when $p^{\prime} \geq d(d+1) q / 2$.

In two dimensions the sharp boundedness result is due to Zygmund [37] who extended earlier work by Fefferman and Stein ([18]) (see also [12], [19] for estimates on more general oscillatory integral operators). Initial results in higher dimensions for the smaller range $1 \leq p<\left(d^{2}+2 d\right) /\left(d^{2}+2 d-2\right)$ are due to Prestini [28], with strict inequality $p^{\prime}>d(d+1) q / 2$ for the local result. For the same range of $p$, Christ [13] showed boundedness on the edge $p^{\prime}=d(d+1) q / 2$. The full range (1.1) has been obtained by Drury [14]. The necessity of the condition $p^{\prime} \geq d(d+1) q / 2$ follows by scaling and the necessity of the condition $1 \leq p<p_{d}$ follows from work by Arkhipov, Chubarikov and Karatsuba [1] (cf. also [24], [2] and [9]).

The first problem considered in this paper is what can be said about estimates for the endpoint $p=q=p_{d}$. In two dimensions $p_{2}=4 / 3$ and Beckner, Carbery, Semmes and Soria [6] showed by a Kakeya set argument that the restricted weak type $4 / 3$ estimate fails; in fact $\mathcal{R}$ does not even

\footnotetext{
J.B. was supported in part by grant R01-2004-000-10055-0 of the Korea Science and Engineering Foundation, D.O. was supported in part by NSF grant DMS-0552041, and A.S. was supported in part by NSF grant DMS-0200186.
} 
map $L^{4 / 3,1}\left(\mathbb{R}^{2}\right)$ to $L^{1, \infty}(I)$. Using a result by Keich [23] this can be further quantified for functions supported in $D_{R}:=\left\{x \in \mathbb{R}^{2}:|x| \leq R\right\}$, namely one gets for large $R$ the lower bound in the equivalence

$$
\sup \left\{\|\mathcal{R} f\|_{L^{1, \infty}(I)}:\|f\|_{L^{4 / 3,1}\left(\mathbb{R}^{2}\right)} \leq 1, \operatorname{supp}(f) \subset D_{R}\right\} \approx[\log R]^{1 / 4} .
$$

The upper bounds can be deduced from the method or results in [19] and indeed the analogue of $(1.2)$ for the $L^{4 / 3}\left(\mathbb{R}^{2}\right) \rightarrow L^{4 / 3}(I)$ inequality holds as well, for functions supported in $D_{R}$ (see also [36] for a related estimate).

In higher dimensions the arguments by Drury are not promising to obtain similar endpoint bounds. He obtained his result by an elegant iteration procedure where it is shown that if the $L^{a} \rightarrow L^{b}$ inequality for the adjoint operator holds on the critical edge for some range $b>b_{0}>q_{d}:=p_{d}^{\prime}=$ $\left(d^{2}+d+2\right) / 2$, then it also holds for $b>\rho\left(b_{0}\right)$ where $b_{0}>\rho\left(b_{0}\right)>q_{d}$ and the sequence recursively defined by $b_{i+1}=\rho\left(b_{i}\right)$ is decreasing and converges to $q_{d}$. We note that the constants in the estimates increase exponentially with the number of iterations, so that a sensible endpoint bound for $\mathcal{R}$ on functions in $L^{p_{d}, 1}$ seems out of reach with this method. Given also the lower bounds (1.2) in two dimensions, it is somewhat surprising that the restricted weak type endpoint bound does hold in three and higher dimensions; in fact the better restricted strong type estimate is true:

Theorem 1.1. Let $d \geq 3$ and $p_{d}=\frac{d^{2}+d+2}{d^{2}+d}$. Then

$$
\mathcal{R}: L^{p_{d}, 1}\left(\mathbb{R}^{d}\right) \rightarrow L^{p_{d}}(\mathbb{R})
$$

is bounded.

Note that all $L^{p} \rightarrow L^{q}$ inequalities for $\mathcal{R}$ can be deduced by interpolation with trivial estimates for $L^{1}$ functions. Arguments in [2] or [9] show that the source space $L^{p_{d}, 1}$ cannot be replaced by a larger Lorentz space. The argument in $\S 5$ below also shows that the target space $L^{p_{d}}$ cannot be replaced by a smaller Lorentz space. It would be interesting to investigate whether the validity of the endpoint bounds in higher dimensions has implications to some integral geometric or Kakeya type problems.

The estimate for $\mathcal{R}$ is deduced from an estimate for the adjoint operator which after some rescaling leads to a problem about more general oscillatory integral operators given by

$$
T_{\lambda} f(x)=\int e^{i \lambda \phi(x, t)} a(x, t) f(t) d t
$$

where $\lambda \gg 1$, the amplitude $a$ is $C^{\infty}$ and compactly supported in $\Omega \times I \subset$ $\mathbb{R}^{d} \times \mathbb{R}$ and $\phi$ is a real valued phase function in $C^{\infty}(\Omega \times I)$. Following [3] we impose the curvature condition that for each $x \in \Omega$ the curve $s \mapsto \nabla_{x} \phi(x, s)$ is nondegenerate, in the sense that

$$
\operatorname{det}\left(\partial_{t}\left(\nabla_{x} \phi\right), \partial_{t}^{2}\left(\nabla_{x} \phi\right), \ldots, \partial_{t}^{n}\left(\nabla_{x} \phi\right)\right) \neq 0
$$

in $\Omega \times I$. 
Theorem 1.2. Suppose that $d \geq 3, \lambda>0$, and that (1.4) holds in $\Omega \times I$. Let $q_{d}=\frac{d^{2}+d+2}{2}$. Then

$$
\left\|T_{\lambda}\right\|_{L^{q_{d}(I) \rightarrow L^{q_{d}, \infty}\left(\mathbb{R}^{d}\right)}} \leq C(1+\lambda)^{-d / q_{d}}
$$

Moreover,

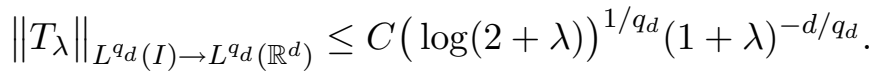

Again (1.5) and even the weaker restricted weak type inequality fail in two dimensions, by a Kakeya set argument ([6], [35]). Calculations with $f \in C_{0}^{\infty}$ and the phase for the extension operator, $\phi(x, t)=-\langle\gamma(t), x\rangle$, show that (1.6) is sharp; moreover the space $L^{q_{d}, \infty}\left(\mathbb{R}^{d}\right)$ in (1.5) cannot be replaced by a smaller Lorentz space $L^{q_{d}, s}$, see [2], [9]. Finally we shall show in $\S 5$ that for the case of the extension operator in (1.5) $L^{q_{d}}$ cannot be replaced by any Lorentz space $L^{q_{d}, \rho}$ with $\rho>q_{d}$. We point out an important aspect of the proof of Theorem 1.2, namely the idea that in order to prove a weak type $(q, q)$ bound for very large $q$ one estimates a multilinear expression with many symmetries on the space $L^{r, \infty}$, for some $r<1$, and takes advantage of the $r$-convexity of this space $(c f . \S 2)$.

We now turn to classes of curves for which the nondegeneracy condition is not satisfied. It has long been known that restriction theorems such as Theorem 1.1 hold under a finite type condition, if $p$ is taken from a sufficiently small interval $[1,1+\epsilon)$ with $\epsilon$ depending on the "type"; for the known results of this type see the papers by Sjölin [30] and Sogge [31] in two dimensions and by Christ [13] and Drury and Marshall [16], [17] in higher dimensions. Another direction that has been pursued is to prove a sharp universal restriction theorem in the full range $p \in\left[1, p_{d}\right)$, on the critical edge $1 / q=d(d+1) /\left(2 p^{\prime}\right)$. Now the standard measure needs to be replaced by the affine arclength measure given by $w(t) d t$ where

$$
\begin{aligned}
w(t) & \equiv w_{\gamma}(t)=|\tau(t)|^{\frac{2}{d(d+1)}}, \\
\tau(t) & \equiv \tau_{\gamma}(t)=\operatorname{det}\left(\gamma^{\prime}(t), \quad \gamma^{\prime \prime}(t), \quad \ldots, \quad \gamma^{(d)}(t)\right) .
\end{aligned}
$$

The objective is then to prove the endpoint inequality

$$
\left(\int_{I}|\widehat{f}(\gamma(t))|^{q} w(t) d t\right)^{1 / q} \leq C\|f\|_{p}, \quad p^{\prime}=\frac{d(d+1)}{2} q,
$$

for all $p<p_{d}$, see e.g. [15] for a general discussion.

Note that the arclength measure is invariant under reparametrization. Moreover, an important feature of the inequality (1.9) on the critical edge is its invariance under general linear change of variables.

In two dimensions, inequality (1.9) has been proved by Sjölin [30] for large classes of convex curves, see also Oberlin [26]. Moreover Drury and Marshall [17] proved a positive result for large classes of finite type curves in higher dimensions, in the partial range $p<\frac{d^{2}+2 d}{d^{2}+2 d-2}$ (i.e. $p<15 / 13$ in 
three dimensions), and Drury [15] improved the result for some curves in three dimensions, obtaining a better partial result for $p<36 / 31$.

It is conceivable that the inequality (1.9) is true for all curves, as to our best knowledge no counterexamples are known. However, in three and higher dimensions, very few positive results have been known for all $p<p_{d}$. For example given the family of monomial curves with nonvanishing curvature, i.e. $\left(t, t^{2}, t^{\beta}\right)$, the only three cases for which (1.9) has been known in the full range $p<p_{3}=7 / 6$ are (i) the trivial case $\beta=2$ where the weight $w$ vanishes identically, (ii) the nondegenerate case $\beta=3$, and, (iii), the curious exceptional case $\beta=9$, which follows via a change of variables from an estimate for "rough" nondegenerate curves, due to the first two authors [4], $c f$. also Theorem 1.4 below.

We prove sharp and uniform $L^{p} \rightarrow L^{q}$ estimates for all monomial curves.

Theorem 1.3. For given real numbers $a_{1}, \ldots, a_{d}, d \geq 2$, consider the curve

$$
t \mapsto \gamma(t)=\left(t^{a_{1}}, t^{a_{2}}, \ldots, t^{a_{d}}\right), \quad 0<t<\infty,
$$

let $w$ be as in (1.7) and suppose $1<p<p_{d}=\frac{d^{2}+d+2}{d^{2}+d}$, and $p^{\prime}=\frac{d(d+1)}{2} q$. Then there is $C(p, d)<\infty$ so that for all $f \in L^{p}\left(\mathbb{R}^{d}\right)$

$$
\left(\int_{0}^{\infty}|\widehat{f}(\gamma(t))|^{q} w(t) d t\right)^{1 / q} \leq C(p, d)\|f\|_{L^{p}\left(\mathbb{R}^{d}\right)} .
$$

It should be emphasized that the finite constant $C(p, d)$ does not depend on the choice of exponents $a_{1}, \ldots, a_{d}$. Some related results for classes of 'simple' curves $\left(t, t^{2}, \ldots, t^{d-1}, \phi(t)\right)$, but with possibly flat $\phi$ will be treated in a subsequent paper [5].

Finally, it is natural to ask whether a restricted strong type estimate with respect to affine arclength measure holds at the endpoint $p=p_{d}, d \geq 3$, for some class of "degenerate curves". This remains largely open, and we have a positive result only for special cases. We formulate such a result for certain curves in $\mathbb{R}^{3}$; note that the critical exponent is $p_{3}=7 / 6$.

Theorem 1.4. Let $\gamma(t)=\left(t, t^{\alpha}, t^{\beta}\right)$, and $w(t)=\left|\operatorname{det}\left(\gamma^{\prime}(t), \gamma^{\prime \prime}(t), \gamma^{\prime \prime \prime}(t)\right)\right|^{1 / 6}$. Then there is a universal constant $C$ so that the inequality

$$
\left(\int_{0}^{\infty}|\widehat{f}(\gamma(t))|^{7 / 6} w(t) d t\right)^{6 / 7} \leq C\|f\|_{L^{7 / 6,1}\left(\mathbb{R}^{3}\right)} .
$$

holds in the following two cases:

(i) $\alpha+\beta=5, \alpha \notin(2,3)$,

(ii) $\beta=5 \alpha-1, \alpha \notin(1 / 3,1 / 2)$.

The proof of this result is a combination of the method in $\S 3$ with ideas in [4].

Structure of the paper: In $\S 2$ we discuss preliminaries on Lorentz spaces, multilinear interpolation and the Drury-Marshall bound on a class of multilinear operators involving Vandermonde determinants (the proof is given in 
an appendix). The weak type estimates for nondegenerate curves are proved in $\S 3$, and the strong type bound (1.6) is proved in $\S 4$. $\S 5$ contains a lower bound for the norms of the extension operator proving the sharpness of the weak type $q_{d}$ bound. Theorem 1.3 is proved in $\S 6$ and Theorem 1.4 in $\S 7$.

Acknowledgement. This paper relies substantially on ideas in the articles by M. Christ [13], S. Drury [14] and by S. Drury and B. Marshall [16], [17]. We have added an exposition of some of their work hoping that the paper becomes more accessible. We also thank the referees for their comments.

\section{Preliminaries}

Lorentz spaces. We use the standard quasi-norm on the Lorentz space $L^{p, q}$, namely for $p, q<\infty$

$$
\|f\|_{p, q}=\left(\frac{q}{p} \int_{0}^{\infty}\left[t^{1 / p} f^{*}(t)\right]^{q} \frac{d t}{t}\right)^{1 / q}, \quad q<\infty
$$

where $f^{*}$ is the nonincreasing rearrangement of $f$. Moreover

$$
\|f\|_{p, \infty}=\sup _{t>0} t^{1 / p} f^{*}(t)=\sup _{\lambda>0} \lambda[\operatorname{meas}(\{x:|f(x)|>\lambda\})]^{1 / p} .
$$

This does not define a norm unless $1 \leq p=q$; however $L^{p, q}$ is normable if $1<p<\infty$ and $1 \leq q \leq \infty$. For this and many other useful properties on Lorentz spaces we refer to [20] or [33].

We state some facts on Lorentz spaces needed later. First, there is the following variant of Hölder's inequality for the $L^{r, \infty}$ quasinorms, namely

$$
\left\|\prod_{i=1}^{n} h_{i}\right\|_{r, \infty} \leq n^{1 / r} \prod_{i=1}^{n}\left\|h_{i}\right\|_{r s_{i}, \infty}, \quad r>0, \sum_{i=1}^{n} \frac{1}{s_{i}}=1 .
$$

This follows by observing that the set $\Omega=\left\{x: \prod_{i=1}^{n}\left|h_{i}\right|>\lambda\right\}$ is contained in the union of the $n$ sets

$$
\Omega_{j}:=\left\{x: \frac{\left|h_{j}(x)\right|}{\left\|h_{j}\right\|_{r s_{j}, \infty}}>\left(\frac{\lambda}{\prod_{i=1}^{n}\left\|h_{i}\right\|_{r s_{i}, \infty}}\right)^{1 / s_{j}}\right\} .
$$

Next, as mentioned in the introduction, we shall use bounds for multilinear operators on $L^{r, \infty}$ for $r<1$. The advantage of working with the spaces $L^{r, \infty}, r<1$ (as opposed to $L^{1, \infty}$, say) is that they are $r$-convex, that is, the inequality

$$
\left\|\sum_{l=1}^{N} h_{l}\right\|_{X} \leq C_{r}\left(\sum_{l=1}\left\|h_{l}\right\|_{X}^{r}\right)^{1 / r}
$$

holds for $X=L^{r, \infty}$ with $C_{r}$ independent of $N$. This is a result of Stein, Taibleson and G. Weiss [32] who prove $(2.2)$ with $C_{r}=\left(\frac{2-r}{1-r}\right)^{1 / r}$; independently the $r$-convexity of $L^{r, \infty}$ was shown by Kalton [22] (who states that Pisier and Zinn also proved an equivalent result). Note that in contrast $L^{1, \infty}$ is not 1-convex, however a useful precursor to (2.2) in this case had been found by Stein and N. Weiss in [34]. 
We note the following immediate consequence of (2.2).

Lemma 2.1. Let $0<r<1, r \leq p<\infty$, and let $Y$ be a complete $r$ convex space of measurable functions. Suppose that the linear operator $S$ maps simple functions to measurable functions in $Y$ and that for every for every measurable set $E$, and for every simple $f$ with $|f(x)| \leq \chi_{E}(x)$ a.e., the inequality $\|S f\|_{Y} \leq C|E|^{1 / p}$ holds. Then $S$ maps $L^{p, r}$ boundedly to $Y$.

In particular we may consider $Y=L^{r, \infty}, r<1$; thus a linear operator $S$ which is restricted weak type $r$ is also of weak type $r$. To verify Lemma 2.1 let $f^{*}$ be the nonincreasing rearrangement of $f$ and let $E_{l}$ be the set of all $x$ for which $f^{*}\left(2^{l}\right)<|f(x)| \leq f^{*}\left(2^{l-1}\right)$; thus the measure of $E_{l}$ does not exceed $2^{l}$. Define $g_{l}(x)=\chi_{E_{l}}(x) f(x) /\left|f^{*}\left(2^{l-1}\right)\right|$ if $E_{l}$ has positive measure (otherwise put $\left.g_{l}=0\right)$. By assumption $\left\|S g_{l}\right\|_{Y} \leq C\left|E_{l}\right|^{1 / p}$. Since $f=\sum_{l} f^{*}\left(2^{l-1}\right) g_{l}$ we get by $(2.2)\|S f\|_{Y} \leq C\left(\sum_{l}\left[f^{*}\left(2^{l-1}\right)\right]^{r}\left|E_{l}\right|^{r / p}\right)^{1 / r} \leq C\left(\sum_{l}\left[2^{l / p} f^{*}\left(2^{l-1}\right)\right]^{r}\right)^{1 / r}$ which implies the assertion of the Lemma.

We shall use an analytic interpolation theorem for Lorentz spaces, for all parameters; this is due to Y. Sagher [29] who extended a version of the RieszThorin theorem for Lorentz spaces by Hunt [20]. These results were proved for all indices using the harmonic majorization of subharmonic functions. We state the following consequence of Sagher's theorem:

Proposition 2.2. Let $T$ be a multilinear map defined on $n$ tuples of simple functions, with values in measurable functions (on some measure space) so that the inequality

$$
\left\|T\left(f_{1}, \ldots, f_{n}\right)\right\|_{r_{i}, s_{i}} \leq A_{i} \prod_{j=1}^{n}\left\|f_{j}\right\|_{p_{j, i}, q_{j, i}}
$$

holds for $i=0,1$. Then there is a constant $C$ depending only on the exponents $r_{i}, s_{i}, p_{j, i}, q_{j, i}$ so that

$$
\left\|T\left(f_{1}, \ldots, f_{n}\right)\right\|_{r, s} \leq C A_{0}^{1-\vartheta} A_{1}^{\vartheta} \prod_{j=1}^{n}\left\|f_{j}\right\|_{p_{j}, q_{j}}
$$

holds for $\left(\frac{1}{p_{j}}, \frac{1}{q_{j}}, \frac{1}{r}, \frac{1}{s}\right)=(1-\vartheta)\left(\frac{1}{p_{j, 0}}, \frac{1}{q_{j, 0}}, \frac{1}{r_{0}}, \frac{1}{s_{0}}\right)+\vartheta\left(\frac{1}{p_{j, 1}}, \frac{1}{q_{j, 1}}, \frac{1}{r_{1}}, \frac{1}{s_{1}}\right)$.

This follows from Sagher's theorem by normalizing each entry $f_{j}$ in $L^{p, q}$, so that $\left\|f_{j}\right\|_{p, q}=1$ and then imbedding each entry in an analytic family $f_{j, z}$, so that $f_{j}=f_{j, \vartheta}, f_{j, z}=e^{i \arg \left(f_{j}\right)} G_{j, 0}^{1-z} G_{j, 1}^{z}$ with suitable $G_{j, 0}, G_{j, 1}$, and $\left\|G_{j, i}\right\|_{p_{i}, q_{i}} \leq C, i=0,1$. See [20] and also [29].

We shall use a version of a multilinear interpolation argument introduced by M. Christ in [13], often referred to as the multilinear trick. The result is summarized in

Proposition 2.3. Let $0<\beta_{j}<\infty, j=1, \ldots, n$, let

$$
H=\left\{x \in \mathbb{R}^{n}: \sum_{j=1}^{n} \beta_{j}^{-1} x_{j}=1\right\},
$$


and let $K$ be a compact subset of $H \cap[0, \infty)^{n}$. Denote by conv $(K)$ the convex hull of $K$ and by $(\text { conv } K)^{o}$ its interior (with respect to the subspace topology on $H$ induced by $\left.\mathbb{R}^{n}\right)$.

Let $r \leq 1$, let $p_{j} \geq r, j=1, \ldots, n$, and let $Y$ be an $r$-convex Lorentz space (i.e. if $r<1$ then $Y=L^{\rho, q}$ for $\rho \in[r, \infty), q \in[r, \infty]$, or $\left.Y=L^{\infty}\right)$. Let $T$ be an $n$-linear map with values in $Y$, defined on $n$-tuples of simple functions, so that

$$
\left\|T\left(f_{1}, \ldots, f_{n}\right)\right\|_{Y} \leq \prod_{j=1}^{n}\left\|f_{j}\right\|_{L^{p_{j}, r},}, \quad\left(p_{1}^{-1}, \ldots, p_{n}^{-1}\right) \in K,
$$

here, $p_{j}=\infty$ is allowed but $L^{\infty, r}$ should be interpreted as $L^{\infty}$.

Then, for $\left(p_{1}^{-1}, \ldots, p_{n}^{-1}\right) \in(\operatorname{conv}(K))^{o}$ and $\sum_{j=1}^{n} q_{j}^{-1}=r^{-1}$,

$$
\left\|T\left(f_{1}, \ldots, f_{n}\right)\right\|_{Y} \leq C \prod_{j=1}^{n}\left\|f_{j}\right\|_{p_{j}, q_{j}} .
$$

For Banach spaces $Y$ this is due to Christ [13]. The version for all Lorentz spaces can be proved using results on analytic interpolation in the form of Proposition 2.2 in combination with Christ's method and Lemma 2.1. We sketch Christ's argument for the case $r<1$. First, Proposition 2.2 yields the inequality $(2.3)$ for all $\left(p_{1}^{-1}, \ldots, p_{n}^{-1}\right) \in \operatorname{conv}(K)$. Next, the main idea is to assume that $\left|f_{1}(x)\right| \leq \chi_{E}(x)$ a.e., and then to prove, for $k=1, \ldots, n$, and all $\left(p_{1}^{-1}, \ldots, p_{n}^{-1}\right)$ in the interior of $\operatorname{conv}(K)$, the inequality

$$
\left\|T\left(f_{1}, \ldots, f_{n}\right)\right\|_{Y} \leq C\left(p_{1}, \ldots, p_{n}\right)|E|^{1 / p_{1}} \prod_{j=2}^{k}\left\|f_{j}\right\|_{p_{j}, \infty} \prod_{j=k+1}^{n}\left\|f_{j}\right\|_{p_{j}, r} ;
$$

with the obvious interpretation that the first product is 1 if $k=1$ and the second product is 1 if $k=n$ (we are interested in this last case). We argue by induction and assume that (2.5) is true for some $k=k_{o}<n$ (the case $k=1$ has been already obtained, in all of $\operatorname{conv}(K)$ ). We freeze $p_{i}$ for $i \notin\left\{1, k_{o}+1\right\}$ and consider the line segment $\ell$ obtained by intersecting $\operatorname{conv}(K)$ with the two-dimensional plane $\left\{x: x_{i}=p_{i}^{-1}, i \neq 1, i \neq k_{o}+1\right\}$. If $X=\left(p_{1}^{-1}, \ldots, p_{d}^{-1}\right)$ is in the interior of $\operatorname{conv}(K)$ then it is in the interior of that line segment. We interpret the inequality (2.5) as a linear operator acting on $f_{k}$ and, by real interpolation (i.e. the Marcinkiewicz theorem in its general form) we get (2.5) for $k=k_{o}+1$ on the open line segment.

Let $\mathfrak{S}^{n}$ be the group of permutation on $n$ letters. Given any $\varpi \in \mathfrak{S}^{n}$ we can apply $(2.5)$ for $k=n$ to the operator $T^{\varpi}$ defined by $T^{\varpi}\left(f_{1}, \ldots, f_{n}\right)=$ $T\left(f_{\varpi(1)}, \ldots, f_{\varpi(n)}\right)$, with $K$ modified appropriately. By using also Lemma 2.1 we get, for $k=1, \ldots, n$,

$$
\left\|T\left(f_{1}, \ldots, f_{n}\right)\right\|_{Y} \leq C^{\prime}\left(p_{1}, \ldots, p_{n}\right)\left\|f_{k}\right\|_{p_{k}, r} \prod_{j \neq k}\left\|f_{j}\right\|_{p_{j}, \infty} .
$$

This is already a special case of the assertion and the general case follows by further multiple applications of Proposition 2.2. 
Remark: Alternatively a more general result can be obtained for LionsPeetre interpolation spaces; an elegant version for $r$-convex quasi-normed spaces which in several respects is more general is due to Janson [21], and Proposition 2.3 can be seen as a special case of his result.

Vandermonde operators. We now discuss a result by Drury and Marshall which concerns certain multilinear operators involving the Vandermonde determinants. For a vector $x \in \mathbb{R}^{d}$ let $V_{d}(x)$ be the determinant of the $d \times d$ Vandermonde matrix $\left(x_{i}^{j-1}\right)_{i, j} ;$ i.e.

$$
V_{d}(x)=\prod_{1 \leq i<j \leq d}\left(x_{j}-x_{i}\right)
$$

For $h=\left(h_{1}, \ldots, h_{d-1}\right) \in\left(\mathbb{R}_{+}\right)^{d-1}$ define $\kappa(h) \in[0, \infty)^{d}$ by

$$
\kappa_{1}(h)=0, \quad \kappa_{j}(h)=h_{1}+\cdots+h_{j-1}, \quad 2 \leq j \leq d,
$$

and

Define

$$
v(h) \equiv v_{d}(h)=V_{d}(\kappa(h)) .
$$

$$
\mathfrak{V}\left[f_{1}, \ldots, f_{d}\right](t, h):=v(h)^{-1} \prod_{i=1}^{d} f_{i}\left(t+\kappa_{i}(h)\right) .
$$

Let $L_{v}^{A}\left(L^{B}\right)$ denote the weighted mixed norm space consisting of functions $(t, h) \mapsto G(t, h)$ with $\|G\|_{L_{v}^{A}\left(L^{B}\right)}=\left(\int\|G(\cdot, h)\|_{B}^{A} v(h) d h\right)^{1 / A}<\infty$; then

$$
\begin{aligned}
\left\|\mathfrak{V}\left[f_{1}, \ldots, f_{d}\right]\right\|_{L_{v}^{A}\left(L^{B}\right)}= & \\
& \left(\int\left(\int \prod\left|f_{i}\left(t+\kappa_{i}(h)\right)\right|^{B} d t\right)^{A / B} v(h)^{1-A} d h\right)^{1 / A} .
\end{aligned}
$$

Proposition 2.4. (cf. [16], [17]).

(i) Let, for $\alpha>0$,

$$
\Omega_{d}(\alpha)=\left\{h \in(0, \infty)^{d-1}: v_{d}(h) \leq \alpha\right\}
$$

and assume $d \geq 2$. Then $\Omega_{d}(\alpha)$ has $(d-1)$-Lebesgue measure $\leq C_{d} \alpha^{2 / d}$.

(ii) Suppose that $1<A<\frac{d+2}{d}, 1<A \leq B<\frac{2 A}{d+2-d A}$, and set $\sigma=$ $2 /(d+2-d A)$. For $\nu=1, \ldots, d$ let $Q_{\nu}$ be the point in $\mathbb{R}^{d}$ for which the $\nu^{\text {th }}$ coordinate is $B^{-1}$ and the other coordinates are equal to $(\sigma A)^{-1}$, and let $\Sigma(A, B)$ be the $d-1$ dimensional closed convex hull of the points $Q_{1}, \ldots, Q_{d}$. Suppose that $\left(p_{1}^{-1}, \ldots, p_{d}^{-1}\right) \in \Sigma(A, B)$. Then

$$
\left\|\mathfrak{V}\left[f_{1}, \ldots, f_{d}\right]\right\|_{L_{v}^{A}\left(L^{B}\right)} \leq C \prod_{i=1}^{d}\left\|f_{i}\right\|_{L^{p_{i}, 1}} .
$$

The proof is given in Appendix $\S \mathrm{A}$. As has been pointed out in [17] the Lorentz spaces $L^{p_{j}, 1}$ can be replaced by larger $L^{p_{j}, q_{j}}$, provided that $\left(p_{1}^{-1}, \ldots, p_{d}^{-1}\right)$ belongs to the interior of $\Sigma(A, B)$ and $\sum_{j=1}^{d} q_{j}^{-1}=1$; this follows from Proposition 2.3. However this improvement of Proposition 2.4 
does not seem to be relevant for the critical estimates on the extension operator.

\section{Proof of Theorem 1.2: the Weak type estimate}

Instead of a single oscillatory integral operator it will be convenient to consider classes of operators with certain uniform estimates, depending on the derivatives of phase and amplitude.

Definition. (i) Let $N \gg d$ be fixed. Let $B \geq 3$, and $0<b \leq 1 / 2$. Denote by $\mathfrak{A}(B)$ the class of functions $a \in C^{N}\left(\mathbb{R}^{d} \times \mathbb{R}\right)$ which are supported in the cylinder $\mathcal{Z}:=\{(x, t):|x| \leq 1,|t| \leq 1\}$ and which satisfy the inequalities

$$
\left|\partial_{x}^{\alpha} \partial_{t}^{j} a(x, t)\right| \leq B,|\alpha| \leq N, j \leq N,
$$

for all $(x, t) \in \mathcal{Z}$

(ii) Let $\mathcal{Z}_{2}:=\{(x, t):|x| \leq 2,|t| \leq 2\}$ and let $\Phi[B, b]$ be the class of phase functions $\phi \in C^{N}$ for which the inequalities

$$
\left|\operatorname{det}\left(\partial_{t}\left(\nabla_{x} \phi\right), \partial_{t}^{2}\left(\nabla_{x} \phi\right), \ldots, \partial_{t}^{n}\left(\nabla_{x} \phi\right)\right)\right| \geq b
$$

and

$$
\left|\partial_{x}^{\alpha} \partial_{t}^{j} \phi(x, t)\right| \leq B, \quad 1 \leq j \leq N, \quad 1 \leq|\alpha| \leq N,
$$

hold, for all $(x, t) \in \mathcal{Z}_{2}$.

(iii) Let

$$
\mathcal{A}_{R}(B, b):=\sup _{\lambda \leq R} \sup \left\{(1+\lambda)^{d / q_{d}}\left\|T_{\lambda} f\right\|_{q_{d}, \infty}\right\}
$$

where the inner supremum is taken over all $f \in L^{q_{d}}$ with $\|f\|_{L^{q_{d}}} \leq 1$ and all oscillatory integral operators $T_{\lambda}$ of the form (1.3) for which the amplitude a belongs to $\mathfrak{A}[B]$ and the phase $\phi$ belongs to $\Phi[B, b]$.

Clearly $\mathcal{A}_{R}(B, b)$ is increasing in $R$ and finite for any choice of $R, B, b$; an immediate estimate is $\mathcal{A}_{R}(B, b)=O\left(R^{d / q}\right)$ as $R \rightarrow \infty$. However we need to prove that

$$
\mathcal{A}_{R}(B, b)=O(1), \quad R \rightarrow \infty,
$$

with the implicit constant only depending on $B, b$. By suitable rescaling arguments one can show some stability of the constants $\mathcal{A}_{R}(B, b)$, namely:

Lemma 3.1. The inequality

$$
\mathcal{A}_{R}(B, b) \lesssim C(B, b)\left(1+\mathcal{A}_{R}\left(3,2^{-1}\right)\right)
$$

holds for $b \leq 1 / 2, B \geq 3$.

The notation $\lesssim$ indicates a constant which does not depend on the parameters $B, b, R$ (but may depend on the dimension). It is easy to see that Lemma 3.1 is equivalent with

$$
\mathcal{A}_{R}(2 B, 2 b) \lesssim \widetilde{C}(B, b)\left(1+\mathcal{A}_{R}(B, b)\right)
$$

for $b \leq 1 / 2, B \geq 3$. We shall first take Lemma 3.1 for granted and give a proof at the end of this section. 
Proof of Theorem 1.2. We need to show that $\mathcal{A}_{R}(B, b)$ in (3.1) is bounded, uniformly in $R$. We may assume that the support of $a$ is in a small ball of radius $\leq\left(b / B^{d}\right)^{1000}$. We fix $R \gg 1$ and we shall estimate the $L^{q_{d}} \rightarrow L^{q_{d}, \infty}$ operator norm $\left\|T_{\lambda}\right\|$ for $\lambda \leq R$, provided that $a \in \mathfrak{A}(B)$ and $\phi \in \Phi(B, b)$. We may assume that $\lambda \geq C_{0}(B, b)$ (suitably chosen).

We now choose an integer $n>q_{d}$ and estimate the $n$-linear expression

$$
\mathfrak{M}_{\lambda}\left(g_{1}, \ldots, g_{n}\right)=\prod_{j=1}^{n} T_{\lambda} g_{j}
$$

observe that $\left\|T_{\lambda} f\right\|_{q_{d}, \infty}=\left\|\mathfrak{M}_{\lambda}(f, \ldots, f)\right\|_{q_{d} / n, \infty}^{1 / n}$. We will take $n=d q_{d}$ (although a similar argument works for any $n>q_{d}$ ). Using the symmetry of $\mathfrak{M}_{\lambda}$ we may factor it in various ways and first derive estimates for the $d$-linear expression

$$
\mathcal{M}_{\lambda}\left(f_{1}, \ldots, f_{d}\right)=\prod_{i=1}^{d} T_{\lambda} f_{i} .
$$

Let $\chi_{k}$ be the indicator function of

$$
S_{k}=\left\{t \in \mathbb{R}^{d}: 2^{-k-1} \leq \prod_{1 \leq i<j \leq d}\left|t_{i}-t_{j}\right|<2^{-k}\right\} .
$$

Following [3] split $\mathcal{M}_{\lambda}=\sum_{k \in \mathbb{Z}} \mathcal{M}_{\lambda, k}$, where

$$
\mathcal{M}_{\lambda, k}\left(f_{1}, \ldots, f_{d}\right)=\int e^{i \lambda\left(\phi\left(x, t_{1}\right)+\ldots \phi\left(x, t_{d}\right)\right)} \prod_{i=1}^{d}\left[a\left(x, t_{i}\right) f_{i}\left(t_{i}\right) \chi_{k}(t)\right] d t_{1} \ldots d t_{d} .
$$

We first use a by now standard $L^{2}$ estimate (for the complete details see [3]). One may introduce the singular change of variables $y=\sum_{i=1}^{d} \phi\left(x, t_{i}\right)$, apply a standard $L^{2}$ estimate for singular integrals and change variables back and it follows that

$$
\begin{aligned}
& \left\|\mathcal{M}_{\lambda, k}\left(f_{1}, \ldots, f_{d}\right)\right\|_{2} \leq \\
& C_{1}(B, b)(1+\lambda)^{-d / 2}\left(\int_{S_{k}} 2^{k}\left|f_{1}\left(t_{1}\right) \cdots f_{d}\left(t_{d}\right)\right|^{2} d t_{1} \cdots d t_{d}\right)^{1 / 2} .
\end{aligned}
$$

The factor $2^{k}$ is comparable to the reciprocal of the Jacobian, which is $\left(\prod_{1 \leq i<j \leq d}\left|t_{i}-t_{j}\right|\right)^{-1}$. The measure of the $t_{1}$-section of $S_{k}$ (i.e. the set of all $\left(t_{2}, \ldots, t_{d}\right)$ for which $\left.\left(t_{1}, \ldots, t_{d}\right) \in S_{k}\right)$ is $O\left(2^{-2 k / d}\right.$ ) (cf. Proposition 2.4, (i)). Thus also

$$
\left\|\mathcal{M}_{\lambda, k}\left(f_{1}, \ldots, f_{d}\right)\right\|_{2} \lesssim C_{1}(B, b) 2^{(d-2) k /(2 d)}(1+\lambda)^{-d / 2}\left\|f_{1}\right\|_{2} \prod_{i=2}^{d}\left\|f_{i}\right\|_{\infty} .
$$

Now let $u\left(h_{1}, \ldots, h_{d-1}\right)=h_{1} \cdots h_{d-1} \prod_{1 \leq i<j \leq d-1}\left|h_{i}-h_{j}\right|$. Then

$$
\mathcal{M}_{\lambda, k}\left(f_{1}, \ldots, f_{d}\right)=\int_{h: 2^{-k-1} \leq u(h) \leq 2^{-k}} \int e^{i \lambda \psi(x, s, h)} \mathfrak{a}(x, s, h) F(s, h) d s d h
$$


where

$$
\begin{gathered}
F(s, h)=f_{1}(s) \prod_{i=1}^{d-1} f_{i+1}\left(s+h_{i}\right), \\
\psi(x, s, h)=\phi(x, s)+\sum_{i=1}^{d-1} \phi\left(x, s+h_{i}\right),
\end{gathered}
$$

and

$$
\mathfrak{a}(x, s, h)=a(x, s) \prod_{i=1}^{d-1} a\left(x, s+h_{i}\right) .
$$

Then it is easy to check that

$$
\mathfrak{a}(\cdot, h) \in \mathfrak{A}(C B), \quad \psi(\cdot, h) \in \Phi\left[C B, C^{-1} b\right],
$$

for some absolute constant $C$, uniformly for the $h$ under consideration.

Define

$$
S_{h, \lambda} F(x)=\int e^{i \lambda \psi(x, t, h)} \mathfrak{a}(x, t, h) F(t) d t
$$

then by the definition of $\mathcal{A}_{R}(C B, b / C)$

$$
(1+\lambda)^{d / q_{d}}\left\|S_{h, \lambda} F\right\|_{q_{d}, \infty} \leq \mathcal{A}_{R}(C B, b / C)\|F\|_{L^{q_{d}}} .
$$

Using the (integral form of the) triangle inequality

$$
\begin{aligned}
(1+\lambda)^{d / q_{d}} \| & \mathcal{M}_{\lambda, k}\left(f_{1}, \ldots, f_{d}\right) \|_{q_{d}, \infty} \\
& \leq \int_{h: 2^{-k-1} \leq u(h) \leq 2^{-k}}(1+\lambda)^{d / q_{d}}\left\|S_{h, \lambda} F(\cdot, h)\right\|_{q_{d}, \infty} d h \\
& \leq \mathcal{A}_{R}(C B, b / C) \int_{h: 2^{-k-1} \leq u(h) \leq 2^{-k}}\|F(\cdot, h)\|_{q_{d}} d h \\
& \leq \mathcal{A}_{R}(C B, b / C) \int_{h: 2^{-k-1} \leq u(h) \leq 2^{-k}}\left\|f_{1}\right\|_{q_{d}} \prod_{i=2}^{d}\left\|f_{i}\right\|_{\infty} d h \\
& \leq C^{\prime} \mathcal{A}_{R}(C B, b / C) 2^{-2 k / d}\left\|f_{1}\right\|_{q_{d}} \prod_{i=2}^{d}\left\|f_{i}\right\|_{\infty} ;
\end{aligned}
$$

here we used again, that meas $\left(\left\{h:|u(h)| \leq 2^{-k}\right\}\right) \approx 2^{-2 k / d}$.

By Lemma 3.1 ( $c f .(3.2))$ we also get

$$
\begin{aligned}
& (1+\lambda)^{d / q_{d}}\left\|\mathcal{M}_{\lambda, k}\left(f_{1}, \ldots, f_{d}\right)\right\|_{q_{d}, \infty} \\
& \quad \leq C_{2}(B, b)\left(1+\mathcal{A}_{R}(B, b)\right) 2^{-2 k / d}\left\|f_{1}\right\|_{q_{d}} \prod_{i=2}^{d}\left\|f_{i}\right\|_{\infty} .
\end{aligned}
$$

Now we interpolate the $L^{2}$ and $L^{q_{d}, \infty}$ bounds (3.4) and (3.5) by the real method (based on the familiar argument by Bourgain [8] for the spherical 
maximal function, see also [11]). Let $\vartheta \in(0,1)$ be defined by

$$
(1-\vartheta) \frac{d-2}{2 d}+\vartheta\left(\frac{-2}{d}\right)=0
$$

then notice that

$$
\vartheta=\frac{d-2}{d+2} \text { and } \frac{1-\vartheta}{2}+\frac{\vartheta}{q_{d}}=\frac{d}{q_{d}}
$$

and $0<\vartheta<1$ if $d \geq 3$.

Thus, for fixed $f_{2}, \ldots, f_{d}$, the linear operator $f_{1} \mapsto \sum_{k} \mathcal{M}_{\lambda, k}\left(f_{1}, \ldots, f_{d}\right)$ is of restricted weak type $\left(q_{d} / d, q_{d} / d\right)$; in fact

$$
\begin{gathered}
\left\|\sum_{k} \mathcal{M}_{\lambda, k}\left(f_{1}, \ldots, f_{d}\right)\right\|_{q_{d} / d, \infty} \leq C_{3}\left[C_{1}(B, b)(1+\lambda)^{-d / 2}\right]^{1-\vartheta} \\
\times\left[(1+\lambda)^{-d / q_{d}}\left(1+\mathcal{A}_{R}(B, b)\right)\right]^{\vartheta}\left\|f_{1}\right\|_{q_{d} / d, 1} \prod_{i=2}^{d}\left\|f_{i}\right\|_{\infty} \\
\leq C_{4}(B, b)(1+\lambda)^{-d^{2} / q_{d}}\left(1+\mathcal{A}_{R}(B, b)\right)^{\vartheta}\left\|f_{1}\right\|_{q_{d} / d, 1} \prod_{i=2}^{d}\left\|f_{i}\right\|_{\infty} .
\end{gathered}
$$

By the symmetry and various interpolations this estimate leads to a restricted weak type (or even improved Lorentz type) estimate for $T_{\lambda}$; however to prove the stronger weak type estimate we now set $n=d q_{d}$ and consider the $n$-linear operator (3.3).

We use (2.1) to bound

$$
\begin{aligned}
& \left\|\prod_{i=1}^{d q_{d}} T_{\lambda} g_{i}\right\|_{1 / d, \infty} \leq C \prod_{k=0}^{q_{d}-1}\left\|\mathcal{M}_{\lambda}\left(g_{k d+1}, \ldots, g_{(k+1) d}\right)\right\|_{q_{d} / d, \infty} \\
& \leq C_{4}(B, b)^{q_{d}}(1+\lambda)^{-d^{2}}\left(1+\mathcal{A}_{R}(B, b)\right)^{\vartheta q_{d}} \prod_{k=0}^{q_{d}-1}\left[\left\|g_{k d+1}\right\|_{q_{d} / d, 1} \prod_{i=2}^{d}\left\|g_{k d+i}\right\|_{\infty}\right] .
\end{aligned}
$$

Using the symmetry of the operator we get for any permutation $\pi$ on $n=d q_{d}$ letters

$$
\left\|\prod_{i=1}^{d q_{d}} T_{\lambda} g_{i}\right\|_{1 / d, \infty} \leq C_{5}(B, b)\left(1+\mathcal{A}_{R}(B, b)\right)^{\vartheta q_{d}}(1+\lambda)^{-d^{2}} \prod_{j=1}^{d q_{d}}\left\|g_{\pi(j)}\right\|_{p_{j}, r_{j}}
$$

where $\left(p_{1}^{-1}, \ldots, p_{d q_{d}}^{-1}\right)$ is in the set

$$
K=\left\{P^{\pi}=\left(P_{1}^{\pi}, \ldots, P_{q_{d}}^{\pi}\right): \pi \in \mathfrak{S}_{d q_{d}}\right\},
$$

with $P^{\pi}$ defined by

$$
P_{\pi(i)}^{\pi}= \begin{cases}d / q_{d}, & i=1, \ldots, q_{d} \\ 0, & q_{d}+1 \leq i \leq d q_{d}\end{cases}
$$


and with $r_{j}=1$ if $1 \leq j \leq q_{d}$ and $r_{j}=\infty, j>q_{d}$ (so that $L^{\infty, \infty}=L^{\infty}$ in (3.7)). The convex hull of the set $K$ is a simplex on the hyperplane $\{X \in$ $\left.\mathbb{R}^{d q_{d}}: \sum_{i=1}^{d q_{d}} X_{i}=d\right\}$ with vertices $P^{\pi}$. Using the multilinear interpolation result of Proposition 2.3, part (ii), we obtain that

$$
\left\|\prod_{i=1}^{d q_{d}} T_{\lambda} g_{i}\right\|_{1 / d, \infty} \leq C_{5}(B, b)\left(1+\mathcal{A}_{R}(B, b)\right)^{\vartheta q_{d}}(1+\lambda)^{-d^{2}} \prod_{j=1}^{d q_{d}}\left\|g_{\pi(j)}\right\|_{p_{j}, s_{j}}
$$

for all $\left(p_{1}^{-1}, \ldots, p_{d q_{d}}^{-1}\right) \in(\operatorname{conv}(K))^{o}$ and $\sum_{j=1}^{d q_{d}} s_{j}^{-1}=r^{-1} \equiv d$. The center $\left(q_{d}^{-1}, \ldots, q_{d}^{-1}\right)$ lies in $(\operatorname{conv}(K))^{o}$ and we can choose $s_{j}=q_{d}$, for $j=$ $1, \ldots, d q_{d}$.

Setting $g_{j}=f$ we get

$$
\begin{aligned}
\left\|T_{\lambda} f\right\|_{q_{d}, \infty}^{d q_{d}} & =\left\|\left[T_{\lambda} f\right]^{d q_{d}}\right\|_{1 / d, \infty} \\
& \leq C_{5}(B, b)\left(1+\mathcal{A}_{R}(B, b)\right)^{\vartheta q_{d}}(1+\lambda)^{-d^{2}}\|f\|_{q_{d}}^{d q_{d}}
\end{aligned}
$$

Thus, by definition of $\mathcal{A}_{R}$,

$$
\mathcal{A}_{R}(B, b) \leq C_{6}(B, b)\left(1+\mathcal{A}_{R}(B, b)\right)^{\vartheta / d}
$$

which gives $\mathcal{A}_{R}(B, b)=O(1)$ as $R \rightarrow \infty$.

Remark: It is perhaps instructive to compare this argument with one in differentiation theory, namely Christ's simplification of the $L^{p}$ boundedness result $(p>1)$ by Nagel, Stein and Wainger [25] on differentiation in lacunary directions. In [25] a bootstrap argument is used which is formally similar to Drury's argument. Our argument resembles the simplification which avoids this iteration, see e.g. Theorem B in [10] for an exposition.

Proof of Lemma 3.1. Let $\phi$ in $\Phi(B, b)$ and let $a \in \mathfrak{A}(B)$. We wish to bound the $L^{q} \rightarrow L^{q, \infty}$ operator norm of $T_{\lambda}$ where

$$
T_{\lambda} f(w)=\int e^{i \lambda \phi(w, s)} a(w, s) f(s) d s .
$$

Let $\chi \in C^{\infty}(\mathbb{R})$ be a function supported in $(-1,1)$ satisfying

$$
\sum_{n \in \mathbb{Z}} \chi(s-n)=1
$$

for all $s \in \mathbb{R}$. The argument will involve rescalings depending on two small numbers $\varepsilon \ll 1$ and $\delta \ll \varepsilon$, in fact we shall see that

$$
\begin{aligned}
& \varepsilon=\left(10(d !)^{2} B^{d} b^{-1}\right)^{-d}, \\
& \delta=10^{-2 N d^{2}}(d !)^{-1}\left(d ! B^{d} b^{-1}\right)^{-N d^{2}}
\end{aligned}
$$

is an admissible choice. 
We begin by observing the trivial estimate

$$
\begin{aligned}
\left\|T_{\lambda}\right\|_{L^{q} \rightarrow L^{q, \infty}} \leq C_{d} \leq C_{d}^{\prime}\left(1+\delta^{-1}\right)^{d / q}(1+\lambda)^{-d / q}, & \\
& \text { if } \lambda \leq \min \left\{\delta^{-1}, R\right\} .
\end{aligned}
$$

This takes care of the case $\lambda \leq \delta^{-1}$, and in what follows we shall assume that $\lambda \geq \delta^{-1}$. We decompose the symbol into pieces supported in $\left(\frac{\delta}{2}, \ldots, \frac{\delta}{2}, \varepsilon\right)$ boxes. To this end we set, for $\mu \in \mathbb{Z}$ and $\nu \in \mathbb{Z}^{d}$,

$$
a_{\mu \nu}(w, s)=a(w, s) \chi_{d}\left(4 \delta^{-1} w-\nu\right) \chi\left(2 \varepsilon^{-1} s-\mu\right)
$$

where $\chi_{d}(w):=\prod_{i=1}^{d} \chi\left(w_{i}\right)$. We also set $s(\mu)=\varepsilon \mu / 2, w(\nu)=\delta \nu / 4, P_{\mu \nu}=$ $(w(\nu), s(\mu))$. Let $J_{\phi}^{\mu \nu}$ be the $d \times d$ matrix with

$$
\left(J_{\phi}^{\mu \nu}\right)_{j k}=\partial_{s}^{j} \partial_{w_{k}} \phi\left(P_{\mu, \nu}\right) .
$$

By assumption $\left|\operatorname{det} J_{\phi}^{\mu \nu}\right| \geq b$. Let $L^{\mu \nu}$ be the inverse matrix of $J_{\phi}^{\mu \nu}$. By the cofactor formula we see that its entries have the bound

$$
\left|\left[L^{\mu \nu}\right]_{j k}\right| \leq(d-1) ! B^{d-1} b^{-1} .
$$

We also denote by $J_{\phi}^{\mu \nu}[\varepsilon]$ the matrix whose $i^{\text {th }}$ row is $\varepsilon^{i} \partial_{s}^{i} \nabla_{w} \phi\left(P_{\mu \nu}\right)$. Let $L^{\mu \nu}[\varepsilon]$ be its inverse so that the $k^{\text {th }}$ column of $L^{\mu \nu}[\varepsilon]$ is equal to $\varepsilon^{-k}$ times the $k^{\text {th }}$ column of $L^{\mu \nu}$. Let $\mathcal{T}_{\lambda}^{\mu \nu}$ be the oscillatory integral operator with phase $\phi$ and amplitude $a_{\mu \nu}$. Then, by the support properties of $a$ and $a_{\mu \nu}$,

$$
\left\|T_{\lambda} f\right\|_{q, \infty} \leq 10^{d+1} \delta^{-d} \varepsilon^{-1} \max _{\mu, \nu}\left\|\mathcal{T}_{\lambda}^{\mu \nu} f\right\|_{L^{q, \infty}}
$$

and it suffices to estimate the individual operators $\mathcal{T}_{\lambda}^{\mu \nu}$.

In what follows we fix $\mu, \nu$ and usually drop the superscripts $\mu, \nu$ in $L^{\mu \nu}$ and $L^{\mu \nu}[\varepsilon]$.

Define a rescaled operator $S_{\Lambda} \equiv S_{\Lambda}^{\mu \nu}$ by

$$
S_{\Lambda} g(x)=\int e^{i \Lambda \psi(x, t)} u(x, t) g(t) d t
$$

where

$$
\psi(x, t)=\delta^{-1} \phi(w(\nu)+L[\varepsilon] \delta x, s(\mu)+\varepsilon t)
$$

and

$$
\begin{aligned}
u(x, t) & \equiv a_{\mu \nu}(w(\nu)+\delta L[\varepsilon] x, s(\mu)+\varepsilon t) \\
& =a(w(\nu)+\delta L[\varepsilon] x, s(\mu)+\varepsilon t) \chi(2 t) \chi_{d}(4 L[\varepsilon] x) .
\end{aligned}
$$

Then

$$
\mathcal{T}_{\lambda}^{\mu \nu} f(w(\nu)+\delta L[\varepsilon] x)=\varepsilon S_{\lambda \delta}[f(s(\mu)+\varepsilon \cdot)](x)
$$

and it follows

$$
\left\|\mathcal{T}_{\lambda}^{\mu \nu}\right\|_{L^{q} \rightarrow L^{q, \infty}} \leq \varepsilon^{1-1 / q}|\operatorname{det} L[\varepsilon]|^{1 / q} \delta^{d / q}\left\|S_{\lambda \delta}\right\|_{L^{q} \rightarrow L^{q, \infty}} .
$$


We verify that $|\operatorname{det} L[\varepsilon]| \leq b^{-1} \varepsilon^{-d(d+1) / 2}$, by the lower bound for $\operatorname{det} J_{\phi}^{\mu \nu}$ and that $\|L[\varepsilon]\|_{\infty} \leq \varepsilon^{-d}(d-1) ! B^{d-1} b^{-1}$; here $\|L[\varepsilon]\|_{\infty}:=\max _{i, j}\left|L_{i j}[\varepsilon]\right|$. We shall then check that

$$
\psi \in \Phi(3,1 / 2)
$$

and

$$
\frac{u}{\|\chi\|_{C^{N}}^{d+1}\left(8 d ! d B^{d} b^{-1} \varepsilon^{-d}\right)^{N}} \in \mathfrak{A}(1) .
$$

Given (3.17) and (3.18) it follows that, for $\delta^{-1} \leq \lambda \leq R$,

$$
\left\|S_{\lambda \delta}\right\|_{L^{q} \rightarrow L^{q, \infty}} \leq(1+\lambda \delta)^{-d / q}\|\chi\|_{C_{N}}^{d+1}\left(8 d ! d B^{d} b^{-1} \varepsilon^{-d}\right)^{N} \mathcal{A}_{R}(3,1 / 2)
$$

and thus, combining this estimate with (3.13) and (3.16) we obtain

$$
\begin{aligned}
& (1+\lambda)^{d / q}\left\|T_{\lambda}\right\|_{L^{q} \rightarrow L^{q, \infty}} \leq \delta^{-d / q}(1+\lambda \delta)^{d / q}\left\|T_{\lambda}\right\|_{L^{q} \rightarrow L^{q, \infty}} \\
& \quad \leq 10^{d+1} \delta^{-d} \varepsilon^{-\left(d^{2}+d+2\right) / 2 q} b^{-1 / q}\|\chi\|_{C^{N}}^{d+1}\left(8 d ! d B^{d} b^{-1} \varepsilon^{-d}\right)^{N} \mathcal{A}_{R}(3,1 / 2) .
\end{aligned}
$$

for $\lambda \geq \delta^{-1}$. If we also take into account the trivial bound (3.11), and the dependence of $\delta$ and $\varepsilon$ on $B$ and $b$ then we get

$$
\mathcal{A}_{R}(B, b) \leq C(B, b, N, d) \mathcal{A}_{R}(3,1 / 2) .
$$

It remains to check (3.17) and (3.18). The latter follows by straightforward applications of the Leibniz rule. Concerning (3.17) we consider the matrix $J_{\psi}$ with entries $\left[J_{\psi}(x, t)\right]_{j k}=\partial_{t}^{j} \psi_{x_{k}}(x, t)$. By definition $J_{\psi}(0,0)$ is the identity matrix. We expand using Taylor's formula

$$
\partial_{t}^{j} \psi_{x_{k}}(0, t)=\sum_{l=0}^{d-j} \frac{t^{l}}{l !} \partial_{t}^{j+l} \psi_{x_{k}}(0,0)+\frac{t^{d-j+1}}{(d-j+1) !} \partial_{t}^{d+1} \psi_{x_{k}}(0, \tilde{t})
$$

and notice that the first sum equals (with $P \equiv P_{\mu \nu}$ )

$$
\sum_{l=0}^{d-j} \frac{t^{l}}{l !} \sum_{m=1}^{d} \partial_{s}^{j+l} \phi_{w_{m}}(P) \varepsilon^{j+l} L_{m k} \varepsilon^{-k}= \begin{cases}0 & \text { if } k<j \\ \frac{t^{k-j}}{(k-j) !} & \text { if } k \geq j .\end{cases}
$$

The absolute value of the remainder term in (3.19) is

$$
\left|\frac{t^{d-j+1}}{(d-j+1) !} \sum_{m=1}^{d} \partial_{s}^{d+1} \phi(\tilde{w}, \tilde{s}) \varepsilon^{d+1} L_{m k} \varepsilon^{-k}\right| \leq 2 d B \varepsilon\|L\|_{\infty} \leq 2 d ! B^{d} b^{-1} \varepsilon .
$$

There is also another error term for the expansion about $x=0$, and we have

$$
\partial_{t}^{j} \psi_{x_{k}}(x, t)-\partial_{t}^{j} \psi_{x_{k}}(0, t)=\sum_{l=1}^{d} \partial_{t}^{j} \psi_{x_{k} x_{l}}(\tilde{x}, t) x_{l}
$$


with

$$
\begin{aligned}
\left|\partial_{t}^{j} \psi_{x_{k} x_{l}}(\tilde{x}, t)\right| & =\left|\delta^{-1} \varepsilon^{j-k-l} \delta^{2} \sum_{m, n=1}^{d} L_{m l} L_{n k} \partial_{s}^{j} \phi_{w_{n} w_{m}}(\tilde{w}, \tilde{s})\right| \\
& \leq \delta^{-1+2} \varepsilon^{j-l-k} B d^{2}\left(b^{-1}(d-1) ! B^{d-1}\right)^{2} \leq \delta \varepsilon^{j-l-k}\left(d ! B^{d} b^{-1}\right)^{2} .
\end{aligned}
$$

Thus, for all $(x, t) \in \mathcal{Z}_{2}$,

$$
\left[J_{\psi}(x, t)\right]_{j k}= \begin{cases}\frac{t^{k-j}}{(k-j) !}+E_{j k}(x, t), & \text { if } k \geq j \\ E_{j k}(x, t), & \text { if } k<j,\end{cases}
$$

with

$$
\left|E_{j k}(x, t)\right| \leq 2 d ! B^{d} b^{-1} \varepsilon+2 \delta \varepsilon^{1-2 d} B\left(b^{-1} d ! B^{d-1}\right)^{2} .
$$

By straightforward considerations using cofactor expansions we see that

$$
\left|\operatorname{det} J_{\psi}(x, t)-1\right| \leq(d !-1) \max _{1 \leq \kappa \leq d} \max _{j k}\left|E_{j k}(x, t)\right|^{\kappa},
$$

and thus, by our choice of $\varepsilon$, we have

$$
\operatorname{det} J_{\psi}(x, t) \geq 1 / 2, \quad(x, t) \in \mathcal{Z}_{2},
$$

moreover, using also our choice of $\delta$ and the assumption $|t| \leq 2$

$$
\left|\partial_{t}^{j} \psi_{x_{k}}(x, t)\right| \leq 3, \quad 1 \leq j \leq N
$$

The above estimates for the second derivatives can be extended in a straightforward manner to higher derivatives and we obtain for $j \leq N$, and multiindices $\alpha=\left(\alpha_{1}, \ldots, \alpha_{d}\right)$ with $|\alpha|:=\sum_{i=1}^{d} \alpha_{i}$ that

$$
\left|\partial_{t}^{j} \partial_{x}^{\alpha} \psi(x, t)\right| \leq \frac{1}{\delta} \varepsilon^{j-\alpha_{1}-2 \alpha_{2}-\cdots-d \alpha_{d}} \delta^{|\alpha|}\left(d ! B^{d} b^{-1}\right)^{|\alpha|} .
$$

Observe that when we have at least two $x$-differentiations then the smallness of $\delta$ can be used. By our choice (3.10)

$$
\left|\partial_{t}^{j} \partial_{x}^{\alpha} \psi(x, t)\right| \leq 2, \quad 1 \leq j \leq N, 2 \leq|\alpha| \leq N,
$$

and it follows from (3.22), (3.23) and (3.24) that $\psi \in \Phi\left[3,2^{-1}\right]$.

The extension operators for nondegenerate curves. The model case for our class of phase functions is $\phi(x, t)=-\langle x, \gamma(t)\rangle$ where $\gamma: I \rightarrow \mathbb{R}^{d}$ is defined on the compact interval $I$ and has the property that for each $t$ the derivatives $\gamma^{\prime}(t), \ldots, \gamma^{(d)}(t)$ are linearly independent. Define the Fourier extension operator

$$
\mathcal{E} f(\xi)=\int_{I} f(t) e^{-i\langle\xi, \gamma(t)\rangle} d t
$$

and let $B(\lambda)$ be a ball in $\mathbb{R}^{d}$ of radius $\lambda$. Then by a change of variable Theorem 1.2 implies that there is $C>0$ so that for all $\alpha>0$

$$
\operatorname{meas}(\{\xi \in B(\lambda):|\mathcal{E} f(\xi)|>\alpha\}) \leq\left[C \alpha^{-1}\|f\|_{q_{d}}\right]^{q_{d}} .
$$


By letting $\lambda \rightarrow \infty$ and using the monotone convergence theorem we see that $\mathcal{E}: L^{q_{d}}(I) \rightarrow L^{q_{d}, \infty}\left(\mathbb{R}^{d}\right)$. A duality argument shows the local version of Theorem 1.1, namely

$$
\|\widehat{f} \circ \gamma\|_{L^{p_{d}(I)}} \leq c_{I}\|f\|_{L^{p_{d}, 1}\left(\mathbb{R}^{d}\right)} .
$$

A nonisotropic scaling using the dilations $x \mapsto\left(u x_{1}, u^{2} x_{2}, \ldots, u^{d} x_{d}\right)$ can be used to deduce the global version of Theorem 1.1.

\section{Proof of the $L^{q_{d}}$ Bound}

We now show (1.6). Recall the bounds for $\mathcal{M}_{\lambda, k}$. As $T_{\lambda}$ has bounded operator norms the estimate (3.4) is wasteful for large $k$ and the term $2^{k(1 / 2-1 / d)}(1+\lambda)^{-d / 2}$ can be replaced by a constant.

Note that for all $k \geq 0$

$$
\left\|\mathcal{M}_{\lambda, k}\left(f_{1}, \ldots, f_{d}\right)\right\|_{q_{d}, \infty} \lesssim(1+\lambda)^{-d / q_{d}} 2^{-2 k / d}\left\|f_{1}\right\|_{q_{d}, 1} \prod_{i=2}^{d}\left\|f_{i}\right\|_{\infty},
$$

which follows from (3.5) since we have already established the restricted weak type bound for $q_{d}$. By real interpolation,

$$
\left\|\mathcal{M}_{\lambda, k}\left(f_{1}, \ldots, f_{d}\right)\right\|_{q_{d} / d, 1} \lesssim(1+\lambda)^{-d^{2} / q_{d}}\left\|f_{1}\right\|_{q_{d}, 1} \prod_{i=2}^{d}\left\|f_{i}\right\|_{\infty},
$$

but there is also the trivial bound

$$
\left\|\mathcal{M}_{\lambda, k}\left(f_{1}, \ldots, f_{d}\right)\right\|_{q_{d} / d, 1} \lesssim(1+\lambda)^{-d \vartheta / q_{d}} 2^{-2 k \vartheta / d}\left\|f_{1}\right\|_{q_{d} / d, 1} \prod_{i=2}^{d}\left\|f_{i}\right\|_{\infty},
$$

with $\vartheta=(d-2) /(d+2)$. Let

$$
N_{\lambda}=10 d^{3} \log \lambda
$$

then certainly by $(4.2)$

$$
\left\|\sum_{k>N_{\lambda}} \mathcal{M}_{\lambda, k}\left(f_{1}, \ldots, f_{d}\right)\right\|_{q_{d} / d, 1} \lesssim(1+\lambda)^{-d^{2} / q_{d}}\left\|f_{1}\right\|_{q_{d} / d, 1} \prod_{i=2}^{d}\left\|f_{i}\right\|_{\infty} .
$$

Furthermore one can show, for $1 \leq \tau \leq \infty$,

$$
\left\|\sum_{0 \leq k \leq N_{\lambda}} \mathcal{M}_{\lambda, k}\left(f_{1}, \ldots, f_{d}\right)\right\|_{q_{d} / d, \tau} \lesssim N_{\lambda}^{1 / \tau}(1+\lambda)^{-d^{2} / q_{d}}\left\|f_{1}\right\|_{q_{d} / d, 1} \prod_{i=2}^{d}\left\|f_{i}\right\|_{\infty} .
$$

This follows from the case $\tau=1$ which holds by (4.1) and the case $\tau=\infty$ which is the restricted weak type estimate that follows from Bourgain's interpolation argument. All together

$$
\left\|\prod_{j=1}^{d} T_{\lambda} f_{j}\right\|_{q_{d} / d, \tau} \lesssim N_{\lambda}^{1 / \tau}(1+\lambda)^{-d^{2} / q_{d}}\left\|f_{1}\right\|_{q_{d} / d, 1} \prod_{i=2}^{d}\left\|f_{i}\right\|_{\infty}
$$


and similar bounds with the $f_{i}$ permuted. We apply this with $\tau=q_{d} / d$ and use the multilinear trick for the $q_{d}$-linear expression $\prod_{j=1}^{q_{d}} T_{\lambda} f_{j}$ on $L^{1}$. We have for all permutations $\pi$ on $q_{d}$ letters

$$
\begin{aligned}
\left\|\prod_{j=1}^{q_{d}} T_{\lambda} f_{j}\right\|_{1} & \leq\left\|\prod_{i=1}^{d} T_{\lambda} f_{\pi(i)}\right\|_{\frac{q_{d}}{d}, 1}\left\|\prod_{j=d+1}^{q_{d}} T_{\lambda} f_{\pi(j)}\right\|_{\frac{q_{d}}{q_{d}-d}, \infty} \\
& \leq\left\|\prod_{i=1}^{d} T_{\lambda} f_{\pi(i)}\right\|_{\frac{q_{d}}{d}, 1} \prod_{j=d+1}^{q_{d}}\left\|T_{\lambda} f_{\pi(j)}\right\|_{q_{d}, \infty} \\
& \lesssim N_{\lambda}(1+\lambda)^{-d}\left\|f_{\pi(1)}\right\|_{q_{d} / d, 1} \prod_{i=2}^{d}\left\|f_{\pi(i)}\right\|_{\infty} \prod_{j=d+1}^{q_{d}}\left\|f_{\pi(j)}\right\|_{q_{d}, 1} .
\end{aligned}
$$

The multilinear interpolation result of Proposition 2.3, for $Y=L^{1}$, yields

$$
\left\|\prod_{j=1}^{q_{d}} T_{\lambda} f_{j}\right\|_{1} \lesssim N_{\lambda}(1+\lambda)^{-d} \prod_{i=1}^{q_{d}}\left\|f_{\pi(i)}\right\|_{p_{i}, r_{i}}
$$

for $\left(p_{1}^{-1}, \ldots, p_{q_{d}}^{-1}\right)$ in a neighborhood of $\left(q_{d}^{-1}, \ldots, q_{d}^{-1}\right)$, satisfying $\sum_{i=1}^{q_{d}} p_{i}^{-1}=$ 1 , and for $\sum_{i=1}^{q_{d}} r_{i}^{-1}=1$. Now $N_{\lambda} \approx \log \lambda$ for $\lambda \geq 2$ and the asserted result follows if we set $p_{i}=r_{i}=q_{d}, f_{i}=f$.

\section{A LOWER BOUND}

We show that the extension operator for the nondegenerate case does not map $L^{q_{d}, r}(I)$ to $L^{q_{d}, \infty}\left(\mathbb{R}^{d}\right)$ for $r>q_{d}$. By the uniform boundedness principle it suffices to consider smooth and compactly supported functions and show that the operator norm is not finite. We may assume that $I=(-1,1)$. By a linear change of variable we may also assume that $\gamma^{(j)}(0)=e_{j}$, for $j=1, \ldots, d$.

Let $\chi$ be a nonnegative $C_{0}^{\infty}$ function supported in $(-1 / 8,1 / 8)$ with $\chi(t)=$ 1 for $t \in(-1 / 10,1 / 10)$. For $n \in \mathbb{N}$ define

$$
\begin{aligned}
& u_{n}(t)=2^{n / q_{d}} \chi\left(2^{n}\left(t-2^{-n}\right)\right), \\
& f_{N}(t)=\sum_{n=N+1}^{2 N} u_{n}(t) .
\end{aligned}
$$

It is easy to see that for $N \geq 2$

$$
\left\|f_{N}\right\|_{L^{q_{d}, r}(I)} \leq C N^{1 / r}
$$

and thus it suffices to show that for large $N$

$$
\left\|\mathcal{E} f_{N}\right\|_{L^{q_{d}}, \infty\left(\mathbb{R}^{d}\right)} \geq C N^{1 / q_{d}} .
$$

In order to achieve this we need the following van der Corput type asymptotics which is taken from Lemma 5.1 in [9]. 
Asymptotics. Let $0<h \leq 1, I=[-h, h], I^{*}=[-2 h, 2 h]$ and let $g \in C^{2}\left(I^{*}\right)$. Suppose that $h \leq 10^{-1}\left(1+\|g\|_{C^{2}\left(I^{*}\right)}\right)^{-1}$ and let $\eta \in C^{1}$ be supported in $I$ and satisfy the bounds

$$
\|\eta\|_{\infty}+\left\|\eta^{\prime}\right\|_{1} \leq A_{0}, \text { and }\left\|\eta^{\prime}\right\|_{\infty} \leq A_{1}
$$

Let $k \geq 2$ and define

$$
I_{\lambda}(\eta, x)=\int \eta(s) \exp \left(i \lambda\left(\sum_{j=1}^{k-2} x_{j} s^{j}+s^{k}+g(s) s^{k+1}\right)\right) d s .
$$

Let $\alpha_{k}=\frac{2}{k} \Gamma\left(\frac{1}{k}\right) \sin \left(\frac{(k-1) \pi}{2 k}\right)$, if $k$ is odd and $\alpha_{k}=\frac{2}{k} \Gamma\left(\frac{1}{k}\right) \exp \left(i \frac{\pi}{2 k}\right)$, if $k$ is even. Suppose that $\left|x_{j}\right| \leq \varepsilon \lambda^{(j-k) / k}, j=1, \ldots, k-2$. Then there is an absolute constant $C$ so that, for $\lambda>2$,

$$
\left|I_{\lambda}(\eta, x)-\eta(0) \alpha_{k} \lambda^{-1 / k}\right| \leq C\left[A_{0} \varepsilon \lambda^{-1 / k}+A_{1} \lambda^{-2 / k}\left(1+\delta_{2, k} \log \lambda\right)\right] ;
$$

here $\delta_{2,2}=1$, and $\delta_{2, k}=0$ for $k>2$.

Proof of (5.3). We shall get good lower bounds for the set where $\left|\mathcal{E} f_{N}\right| \geq \beta$ provided that $\beta \ll 2^{-2 N}$. Consider large $\xi$ with $\xi_{d} \approx|\xi|$. By the implicit function theorem the equation $\left\langle\gamma^{(d-1)}(t), \xi\right\rangle=0$ has a unique solution $t_{\mathrm{cr}}(\xi)$ which is homogeneous of degree zero.

For each $n \in[N, 2 N]$ we let $\lambda_{n}=2^{n d / q_{d}} \beta^{-d}$ and set

$$
\begin{aligned}
\mathcal{V}_{n}=\left\{\xi:\left|\xi^{\prime}\right| \leq c\left|\xi_{d}\right|, \lambda_{n} \leq\left|\xi_{d}\right| \leq 2 \lambda_{n}, t_{\mathrm{cr}}(\xi) \in\left(\frac{9}{10} 2^{-n}, \frac{11}{10} 2^{-n}\right),\right. \\
\left.\left|\left\langle\gamma^{(j)}\left(t_{\mathrm{cr}}(\xi)\right), \xi\right\rangle\right| \leq \varepsilon \lambda_{n}^{j / d}, j=1, \ldots, d-2\right\} .
\end{aligned}
$$

Note that if $t \in \operatorname{supp}\left(u_{k}\right)$ and $k \neq n$ then $\left|t-t_{\mathrm{cr}}(\xi)\right| \geq c 2^{-n}$ and therefore $\left|\left\langle\gamma^{(d-1)}(t), \xi\right\rangle\right| \geq c^{\prime} 2^{-n}|\xi|$. By van der Corput's lemma with $(d-1)$ derivatives we get the bound

$$
\left|\mathcal{E} u_{k}(\xi)\right| \leq C 2^{n / q_{d}} 2^{n /(d-1)} \lambda_{n}^{-1 /(d-1)}, \quad \xi \in \mathcal{V}_{n}, \quad k \neq n
$$

By the asymptotics above, if $\varepsilon>0$ is sufficiently small, then

$$
\left|\mathcal{E} u_{n}(\xi)\right| \geq c 2^{n / q_{d}} \lambda_{n}^{-1 / d}-2^{2 n / q_{d}} \lambda_{n}^{-2 / d} \geq c^{\prime} 2^{n / q_{d}} \lambda_{n}^{-1 / d}=c^{\prime} \beta, \quad \xi \in \mathcal{V}_{n} .
$$

Combining the last two inequalities we obtain

$$
\left|\mathcal{E} f_{N}(\xi)\right| \geq c^{\prime \prime} \beta, \quad \xi \in \cup_{n=N}^{2 N} \mathcal{V}_{n}
$$

The measure of $\mathcal{V}_{n}$ is $\geq c_{\varepsilon} 2^{-n} \lambda_{n}^{\frac{(d-2)(d-1)}{2 d}+2}=c_{\varepsilon} 2^{-n} \lambda_{n}^{q_{d} / d}=c_{\varepsilon} \beta^{-q_{d}}$ and the sets $\mathcal{V}_{n}, N \leq n \leq 2 N$ are disjoint if $N$ is large. Thus for $N$ large (5.3) follows. 


\section{Proof of Theorem 1.3}

We first note that it suffices to assume that the powers $b_{i}$ are mutually distinct and also $b_{i} \neq 0$; in the other cases the weight vanishes identically.

We only need to prove the result for $I=(0,1]$ here, by a scaling argument we can easily extend the result to $I=(0, \infty)$, using the linear isomorphisms $x \mapsto\left(s^{b_{1}} x_{1}, \ldots, s^{b_{d}} x_{d}\right)$. Following [17] we will use the exponential parametrization, replacing $t$ by $e^{-t}$. Setting $a_{i}=-b_{i}$ we may assume, after a further linear change of variables, that

$$
\gamma(t)=\left(a_{1}^{-1} e^{a_{1} t}, \cdots, a_{d}^{-1} e^{a_{d} t}\right), \quad 0<t<\infty,
$$

where the $a_{j}$ are real numbers so that either (i) $a_{1}<\cdots<a_{d}<0$, or (ii) $0<a_{1}<\cdots<a_{d}$, or (iii) $a_{1}<\cdots<a_{m}<0<a_{m+1}<\cdots<a_{d}$, for some $m \in\{1, \cdots, d\}$. We shall give the argument for case (iii), and the proofs for the other cases require only notational changes.

Fix any point $(1 / p, 1 / q)$ on the critical line segment $1 / p+\left(d^{2}+d\right) /(2 q)=1$, $0<1 / q<1 / q_{d}$, where $q_{d}=\left(d^{2}+d+2\right) / 2$. Let us fix a number $R>1$ and set $I_{R}=[0, R]$ and let

$$
T_{R} f(x)=\int_{0}^{R} f(t) w(t) e^{-i\langle x, \gamma(t)\rangle} d t .
$$

It suffices to show

$$
\left\|T_{R} f\right\|_{L^{q}\left(\mathbb{R}^{d}\right)} \leq C\|f\|_{L^{p}(w d t)}
$$

with a constant $C$ independent of $R>1$. We need to prove this for $2<p<$ $q_{d}, q=d(d+1) p^{\prime} / 2$ (and $q_{d}>2$ if $d \geq 3$ ); the estimate for $p \leq 2$ follows then by interpolation with the trivial case $p=1$.

Observe that (6.2) holds with some $C=C(a, R)<\infty$, by the estimates for the nondegenerate curve $\gamma$ (restricted to $I_{R}$ ); notice that indeed $|w(t)| \geq$ $C(a) \min \left\{1, e^{R\left(\sum_{j} a_{j}\right) 2 /\left(d^{2}+d\right)}\right\}>0$ on $I_{R}$. Let now $\mathcal{B}_{R, a}$ be the infimum over all $C$ for which (6.2) holds. $\mathcal{B}_{R, a}$ is finite and we have to establish that $\mathcal{B}_{R, a}$ is uniformly bounded in $R \geq 1$ and $a=\left(a_{1}, \ldots, a_{d}\right)$.

We shall estimate the $d$-linear expression

$$
\prod_{j=1}^{d} T_{R} f_{j}(x)=\int_{I_{R}^{d}} e^{i\left\langle x, \gamma\left(t_{1}\right)+\cdots+\gamma\left(t_{d}\right)\right\rangle} \prod_{j=1}^{d}\left[f_{j}\left(t_{j}\right) w\left(t_{j}\right)\right] d t_{1} \cdots d t_{d}
$$

We change variables $\kappa_{j}(h)=\sum_{i=1}^{j-1} h_{i}$ as in $(2.7)$, and let $J_{R}$ denote the set of all $h \in[0, R]^{d-1}$ satisfying $\kappa_{d}(h) \leq R$. For $h \in J_{R}$ let $I_{R, h}=\left[0, R-\kappa_{d}(h)\right]$, and define for any permutation $\pi$ on $d$ letters

$$
F^{\pi}(h, t)=\chi_{J_{R}}(h) \chi_{I_{R, h}}(t) \prod_{i=1}^{d} f_{\pi(i)}\left(t+\kappa_{i}(h)\right) .
$$


For fixed $h$ let

$$
\Gamma(t, h)=\sum_{j=1}^{d} \gamma\left(t+\kappa_{j}(h)\right)
$$

and

$$
H(t, h)=\prod_{j=1}^{d} w\left(t+\kappa_{j}(h)\right) .
$$

Define an operator $S_{R, h}$ by

$$
S_{R, h}[F](x)=\chi_{J_{R}}(h) \int_{I_{R, h}} e^{i\langle x, \Gamma(t, h)\rangle} F(t, h) H(t, h) d t .
$$

Then

$$
\prod_{j=1}^{d} T_{R} f_{j}(x)=\sum_{\pi \in \mathfrak{S}^{d}} \int S_{R, h}\left[F^{\pi}\right](x) d h .
$$

We first give an estimate on the operators $S_{R, h}$ which will put us in the position to apply the Vandermonde estimate (2.10).

Proposition 6.1. Fix $1<p<q_{d}=\frac{d^{2}+d+2}{2}$ and let $q=\frac{d(d+1)}{2} p^{\prime}$. For $\vartheta \in(0,1)$ define

$$
\begin{array}{ll}
\frac{1}{A}=1-\frac{\vartheta}{2}, & \frac{1}{B}=\frac{1}{p}+\vartheta\left(\frac{1}{2}-\frac{1}{p}\right), \\
\frac{1}{s}=\frac{1-\vartheta}{q}+\frac{\vartheta}{2}, & \eta=1-\frac{d+1}{2 q}(1-\vartheta) .
\end{array}
$$

Then (with $v$ as in (2.8))

$$
\begin{aligned}
& \left\|\int S_{R, h}[F] d h\right\|_{s} \\
& \quad \leq C \mathcal{B}_{R, a}^{1-\vartheta}\left(\int\left(\int\left|F(t, h) H(t, h)^{\eta-\frac{d+1}{4} \vartheta}\right|^{B} d t\right)^{\frac{A}{B}} v(h)^{1-A} d h\right)^{\frac{1}{A}} .
\end{aligned}
$$

Proof. The proof relies on arguments in the papers by Drury and Marshall [16], [17]. We begin with a few remarks on the affine arclength measure for the curve $\gamma$ and for the "offspring" curves $t \mapsto \Gamma(t, h)$. Let $\tau$ and $w$ be as in (1.8), (1.7) (for the curve $\gamma$ in (6.1)). Then

$$
|\tau(t)|=v(a) \exp \left(t \sum_{i=1}^{d} a_{i}\right)
$$

with $a=\left(a_{1}, \ldots, a_{d}\right)$, and

$$
H(t, h)^{1 / d}=w(t) \exp \left(\frac{2}{d^{2}(d+1)}\left(\sum_{i=1}^{d} a_{i}\right)\left(\sum_{j=2}^{d} \kappa_{j}(h)\right)\right) .
$$


Next, $\Gamma(t, h)=\gamma(t) E(h)$, where $E(h)$ is a $d \times d$ diagonal matrix with the diagonal entries

$$
E_{i i}(h)=\sum_{j=1}^{d} e^{a_{i} \kappa_{j}(h)}
$$

so that $1 \leq E_{i i}(h) \leq d$, for $1 \leq i \leq m$ and $e^{a_{i} \kappa_{d}(h)} \leq E_{i i}(h) \leq d e^{a_{i} \kappa_{d}(h)}$, for $m+1 \leq j \leq d$ (for the definition of $m$ see the paragraph after (6.1)). Moreover, if $\tau_{h}$ is the expression (1.8) for the curve $\Gamma(\cdot, h)$ then

$$
\tau_{h}(t)=v(a) \exp \left(t \sum_{i=1}^{d} a_{i}\right) \prod_{k=1}^{d} E_{k k}(h)
$$

We first establish the inequality

$$
\| S_{R, h}\left[F H^{\left.-\frac{d-1}{d}\right]} \|_{q} \leq C \mathcal{B}_{R, a}\left(\int|F(t, h)|^{p} H(t, h)^{1 / d} d t\right)^{1 / p}\right.
$$

with a constant $C$ uniform in $h$. Notice that the quotient of $H(t, h)^{1 / d}$ and $w_{h}:=\tau_{h}^{2 /\left(d^{2}+d\right)}$ is independent of $t$, namely

$$
Q(h):=\frac{H(t, h)^{1 / d}}{w_{h}(t)}=\frac{\exp \left(\frac{2}{d^{2}(d+1)}\left(\sum_{j=1}^{d} a_{j}\right)\left(\sum_{j=2}^{d} \kappa_{j}(h)\right)\right)}{\left(\prod_{i=1}^{d}\left(\sum_{j=1}^{d} e^{a_{i} \kappa_{j}(h)}\right)\right)^{\frac{2}{d(d+1)}}} .
$$

Since $\Gamma(t, h)=\gamma(t) E(h)$ we have by affine invariance

$$
\left(\int\left|\int_{I_{R, h}} e^{i\langle x, \Gamma(t, h)\rangle} g(t) w_{h}(t) d t\right|^{q} d x\right)^{1 / q} \leq \mathcal{B}_{R, a}\left(\int|g(t)|^{p} w_{h}(t) d t\right)^{1 / p}
$$

and thus with $g(t):=F(t, h)$,

$$
\left\|S_{R, h}\left[F H^{-(d-1) / d}\right]\right\|_{q} \leq \mathcal{B}_{R, a} Q(h)^{1-1 / p}\left(\int|F(t, h)|^{p} H(t, h)^{1 / d} d t\right)^{1 / p} .
$$

Thus, the estimate (6.9) will follow once we establish the inequality that $Q(h)$ is bounded. But note that

$$
\begin{aligned}
Q(h)^{d(d+1) / 2} & \leq \exp \left(\frac{1}{d}\left(\sum_{j=1}^{m} a_{j}\right)\left(\sum_{j=2}^{d} \kappa_{j}(h)\right)\right) \frac{\exp \left(\frac{1}{d}\left(\sum_{j=m+1}^{d} a_{j}\right)\left(\sum_{j=1}^{d} \kappa_{j}(h)\right)\right)}{\prod_{i=m+1}^{d} e^{a_{i} \kappa_{d}(h)}} \\
& \leq \exp \left(\frac{1}{d}\left(\sum_{j=m+1}^{d} a_{j}\right)\left(\sum_{j=1}^{d}\left(\kappa_{j}(h)-\kappa_{d}(h)\right)\right)\right) \leq 1
\end{aligned}
$$

since $\kappa_{d} \geq \kappa_{d-1} \geq \kappa_{2} \geq \kappa_{1}=0$ and $a_{i}<0$ for $i \leq m, a_{i}>0$ for $i>m$. Thus (6.9) is proved.

We may replace $F$ by $F H^{(d-1) / d}$ and integrate the resulting estimate with respect to $h$. This yields

$$
\int\left\|S_{R, h}[F]\right\|_{q} d h \leq C \mathcal{B}_{R, a} \int\left(\int\left|F(t, h) H(t, h)^{\frac{d-1}{d}+\frac{1}{d p}}\right|^{p} d t\right)^{1 / p} d h .
$$

Note that this implies the claimed estimate (6.8) for the case $\vartheta=0$. 
Now as in [16], [17] one can perform the change of variables $(t, h) \mapsto \Gamma(t, h)$ (justified in [17], p. 549) and use Plancherel's theorem, to obtain

$$
\left\|\int S_{R, h}[F] d h\right\|_{2} \leq C\left(\iint\left|F(t, h) H(t, h) J(t, h)^{-1 / 2}\right|^{2} d t d h\right)^{1 / 2}
$$

where $J(t, h)$ is the Jacobian of this transformation.

Interpolating these two estimates gives

$$
\begin{aligned}
& \left\|\int S_{R, h}[F] d h\right\|_{s} \\
& \leq C \mathcal{B}_{R, a}^{1-\vartheta}\left(\int\left(\int\left|F(t, h) H(t, h)^{\eta} J(t, h)^{-\vartheta / 2}\right|^{B(\vartheta)} d t\right)^{A(\vartheta) / B(\vartheta)} d h\right)^{1 / A(\vartheta)}
\end{aligned}
$$

where $0 \leq \vartheta \leq 1$ and $A, B, s, \eta$ are as in (6.7).

We now use a crucial estimate concerning the determinant of the $d \times d$ matrix $\mathcal{E}(a, s):=\left(e^{a_{i} s_{j}}\right)_{i, j=1, \ldots d}$.

A total positivity bound by Drury and Marshall. [17], p.546. The estimate

$$
\frac{\operatorname{det} \mathcal{E}(a, s)}{\prod_{1 \leq i<j \leq d}\left(\left(a_{j}-a_{i}\right)\left(s_{j}-s_{i}\right)\right)} \geq c_{d} \exp \left(\frac{1}{d}\left(\sum_{j=1}^{d} a_{j}\right)\left(\sum_{j=1}^{d} s_{j}\right)\right)
$$

holds for all real $a_{1}, \ldots, a_{d}$ and all real $s_{1}, \ldots, s_{d}$ with a constant $c_{d}$ that depends only on the dimension $d$.

This means $J(t, h) \geq c_{d} v(h) H(t, h)^{(d+1) / 2}$ and therefore

$$
\begin{aligned}
&\left\|\int S_{R, h}[F] d h\right\|_{s} \leq C\left(\mathcal{B}_{R, a}\right)^{1-\vartheta} \times \\
&\left(\int\left(\int\left|F(t, h) H(t, h)^{\eta-\frac{d+1}{4} \vartheta}\right|^{B} d t\right)^{A / B} v(h)^{-\vartheta A / 2} d h\right)^{1 / A} .
\end{aligned}
$$

Now observe that $A^{-1}=1-\vartheta / 2$ means $-\vartheta A / 2=1-A$ and thus the proof of the proposition is complete.

Proof of Theorem 1.3, continued. Proposition 6.1 enables us to apply the inequality (2.10). We wish to use it for the value

$$
\vartheta=\vartheta(p)=\frac{4(d-1)}{(d+1) d p^{\prime}-4}=\frac{2(d-1)}{q-2}
$$

and we let $A=A_{p}, B=B_{p}, s=s_{p}$ and $\eta=\eta_{p}$ be the values which correspond to $\vartheta=\vartheta(p)$ via (6.7). The reason for this choice is that the exponent of $H$ in (6.8) becomes

$$
\eta_{p}-\frac{d+1}{4} \vartheta=\frac{1}{p}
$$


moreover

$$
s_{p}=\frac{q}{d}=\frac{d+1}{2} p^{\prime} .
$$

In order to apply (2.10) we need the additional restriction $1<A_{p}<\frac{d+2}{d}$, which corresponds to $\vartheta(p)<4 /(d+2)$. A short calculation reveals that this requirement is equivalent with our assumption $p<\frac{d^{2}+d+2}{2}$.

We also set $\sigma_{p}=2 /\left(d+2-d A_{p}\right)$ and obtain after a short computation

$$
\frac{1}{A_{p} \sigma_{p}}=\frac{d+2}{A_{p}}-\frac{d}{2}=\frac{p^{-1}-q^{-1}}{1-2 q^{-1}}
$$

and

$$
\frac{1}{B_{p}}=\frac{1}{p}+\frac{\left(\frac{1}{2}-\frac{1}{p}\right) \frac{d-1}{d p^{\prime}}}{\frac{d+1}{4}-\frac{1}{d p^{\prime}}} .
$$

We check that $B^{-1}>p^{-1}>\left(A_{p} \sigma_{p}\right)^{-1}$ since $2<p$ and we have

$$
\frac{d-1}{A_{p} \sigma_{p}}+\frac{1}{B_{p}}=\frac{d}{p}
$$

Now let $\Sigma\left(A_{p}, B_{p}\right)$ be the simplex defined in the statement of Proposition 2.4. We apply this proposition to the right hand side of (6.8) with $F=F^{\pi}$ as in (6.3); then by (6.6)

$$
\left\|\prod_{j=1}^{d} T_{R} f_{j}\right\|_{q / d} \leq C \mathcal{B}_{R, a}^{1-\vartheta(p)} \prod_{j=1}^{d}\left\|f_{j} w^{1 / p}\right\|_{p_{j}, 1},
$$

for all $\left(p_{1}^{-1}, \ldots, p_{d}^{-1}\right) \in \Sigma\left(A_{p}, B_{p}\right)$.

We continue to argue as in the proof of Theorem 1.2 and consider now the $q_{d}$-linear expression

$$
\mathfrak{M}_{R}\left[g_{1}, \ldots, g_{q_{d}}\right]=\prod_{k=1}^{q_{d}} T_{R}\left[g_{j} w^{-1 / p}\right] .
$$

Consider the set $K_{p}$ consisting of the points $P^{\varpi}=\left(P_{1}^{\varpi}, \ldots, P_{q_{d}}^{\varpi}\right), \varpi \in \mathfrak{S}_{q_{d}}$ (i.e. a permutation on $\left\{1, \ldots, q_{d}\right\}$ ) with $P^{\varpi}$ defined by

$$
P_{\varpi(i)}^{\varpi}= \begin{cases}1 / B_{p}, & i=1, \\ 1 /\left(A_{p} \sigma_{p}\right), & 2 \leq i \leq d, \\ 1 / p, & d+1 \leq i \leq q_{d} .\end{cases}
$$

The (closed) convex hull of $K_{p}$ is a simplex on the hyperplane $\left\{X \in \mathbb{R}^{q_{d}}\right.$ : $\left.\sum_{i=1}^{q_{d}} X_{i}=q_{d} / p\right\}$ with vertices $P^{\varpi}$, and center $\left(p^{-1}, \ldots, p^{-1}\right)$.

By (6.18) and Hölder's inequality

$$
\left\|\mathfrak{M}_{R}\left[g_{1}, \ldots, g_{q_{d}}\right]\right\|_{q / q_{d}} \leq C\left(\mathcal{B}_{R, a}\right)^{(1-\vartheta) q_{d} / d} \prod_{k=1}^{q_{d}}\left\|g_{k}\right\|_{p_{k}, 1}
$$

for all $\left(p_{1}^{-1}, \ldots, p_{q_{d}}^{-1}\right) \in K_{p}$. We now apply Proposition 2.3 and observe that since $q>q_{d}$ our multilinear operator takes values in a Banach space. 
Thus we get

$$
\left\|\mathfrak{M}_{R}\left[g_{1}, \ldots, g_{q_{d}}\right]\right\|_{q / q_{d}} \leq C\left(\mathcal{B}_{R, a}\right)^{(1-\vartheta) q_{d} / d} \prod_{k=1}^{q_{d}}\left\|g_{\varpi(k)}\right\|_{p_{k}, q_{d}},
$$

for all $\left(p_{1}^{-1}, \ldots, p_{q_{d}}^{-1}\right) \in\left(\operatorname{conv} K_{p}\right)^{o}$. Clearly the center $\left(p^{-1}, \ldots, p^{-1}\right)$ belongs to $\left(\operatorname{conv} K_{p}\right)^{o}$ and it follows that for $g_{i}=f w^{1 / p}$,

$$
\left\|T_{R} f\right\|_{q}=\left\|\mathfrak{M}_{R}\left[g_{1}, \ldots, g_{q_{d}}\right]\right\|_{q / q_{d}}^{1 / q_{d}} \leq C(p, d)\left(\mathcal{B}_{R, a}\right)^{(1-\vartheta) / d}\left\|f w^{1 / p}\right\|_{p, q_{d}} .
$$

By $p<q_{d}$ and the continuous imbedding $L^{p} \subset L^{p, q_{d}}$ we have

$$
\left\|f w^{1 / p}\right\|_{L^{p, q_{d}}} \leq\left\|f w^{1 / p}\right\|_{L^{p}}=\left(\int|f(t)|^{p} w(t) d t\right)^{1 / p} .
$$

Thus $\mathcal{B}_{R, a} \leq C^{\prime}(p, d)\left(\mathcal{B}_{R, a}\right)^{(1-\vartheta) / d}$ and the assertion of the theorem follows.

\section{Proof of Theorem 1.4}

Let $\alpha<\beta, \alpha, \beta \notin\{0,1\}$. We first note that the affine arclength measure $w(t) d t$ for the curve $\left(t, t^{\alpha}, t^{\beta}\right), t>0$, is given via $w(t)=c(\alpha, \beta) t^{(\alpha+\beta-5) / 6}$ with $c(\alpha, \beta)^{6}=\alpha \beta(\alpha-1)(\beta-1)(\beta-\alpha)$. We consider the case $\alpha+\beta=5$ which clearly plays a special role as the affine arclength measure is now a constant multiple of Lebesgue measure on $\mathbb{R}$. The case $\alpha=2, \beta=3$ has been handled in $\S 3$, and part (i) of Theorem 1.4 asserts that it holds also true for $\alpha=5-\beta<2$.

To prove this assertion we consider a more general class of curves

$$
t \mapsto(t, y(t), z(t)), t \in I=(0, b) ; \quad 0<b<\infty
$$

where $y, z \in C^{3}(I)$ and satisfy a strong nondegeneracy condition introduced in [4], namely

$$
\Delta(s, t):=\left|y^{\prime \prime}(s) z^{\prime \prime \prime}(t)-y^{\prime \prime \prime}(s) z^{\prime \prime}(t)\right| \geq \delta>0, \quad s, t, \in I ;
$$

moreover it is assumed that

$$
z^{\prime \prime \prime}(t) \neq 0, \quad t \in(0, b),
$$

however no upper bounds for the third derivatives are required on the open interval $(0, b)$. Note that the determinant in (7.2) cannot change sign. In particular, if $h_{1}, h_{2} \geq 0, h_{1}+h_{2}<b$, and if we consider the offspring curves $\Gamma(t, h)=\frac{1}{3} \sum_{i=1}^{3} \gamma\left(t+\kappa_{i}(h)\right), t<b-h_{1}-h_{2}$, then $\Gamma(t, h)=\left(t, y_{h}(t), z_{h}(t)\right)$ where $\left(y_{h}, z_{h}\right)$ satisfies $(7.2)$ (with the same $\left.\delta\right)$ on the interval $\left(0, b-h_{1}-h_{2}\right)$. This follows from an expansion using the multilinearity of the determinant.

Let

$$
\mathcal{E} f(x):=\int_{0}^{b} e^{-i\langle x, \gamma(t)\rangle} f(t) d t .
$$

Proposition 7.1. Let $\gamma$ be as in (7.1), (7.2). Then

$$
\|\mathcal{E} f\|_{L^{7, \infty}\left(\mathbb{R}^{3}\right)} \leq C \delta^{-1 / 7}\|f\|_{L^{7}(I)}
$$


Proof. Let $\mathcal{K}(b, \delta)$ be the class of curves $\gamma$ satisfying $(7.1),(7.2)$ on $(0, b)$ and let

$$
\mathcal{A}_{\delta}(b, R):=\sup _{\rho>0}\left(1+R^{-1} \rho\right)^{-2 d} \sup _{\substack{\gamma \in \mathcal{K}(a, \delta) \\ 0<a \leq b}} \sup _{\|\|_{L^{7}(0, a)} \leq 1}\|\mathcal{E} f\|_{L^{7, \infty}\left(B_{R}\right)^{\circ}}
$$

Clearly $\mathcal{A}_{\delta}(a, R) \leq C(b, R)<\infty$ for $a \leq b$ and we need to show that $\mathcal{A}_{\delta}(a, R)$ is uniformly bounded in $a$ and $R$.

Now let $a \leq b$ and let $\gamma \in \mathcal{K}(a, \delta)$. As in $\S 1.2$ we estimate the trilinear expression $\mathcal{M}\left(f_{1}, f_{2}, f_{3}\right)=\prod \mathcal{E} f_{i}(x)$ and split $\mathcal{M}=\sum_{k} \mathcal{M}_{k}$ where

$$
\mathcal{M}_{k} f(x)=\int_{S_{k}} e^{-i\left\langle x, \sum_{i=1}^{3} \gamma\left(t_{i}\right)\right\rangle} \prod f_{i}\left(t_{i}\right) d t_{1} d t_{2} d t_{3}
$$

with $S_{k}=\left\{\left(t_{1}, t_{2}, t_{3}\right): 2^{-k-1}<V_{3}(t) \leq 2^{-k}\right\}$.

It was observed in Lemma 2 of [4] that the map $\left(t_{1}, t_{2}, t_{3}\right) \rightarrow \frac{1}{3} \sum_{i=1}^{3} \gamma\left(t_{i}\right)$ is one-to-one, when restricted to $\left\{t_{1}<t_{2}<t_{3}\right\}$ (this uses (7.3)). Denote the Jacobian of this mapping by $J\left(t_{1}, t_{2}, t_{3}\right)$. Also as in [14], [4] one uses a generalized mean value theorem $([27]$, V.1.95) to obtain the inequality $J \geq \delta V$. As before this leads to the $L^{2}$ bound

$$
\left\|\mathcal{M}_{k}\left(f_{1}, f_{2}, f_{3}\right)\right\|_{2} \leq C \delta^{-1 / 2} 2^{k / 6}\left\|f_{1}\right\|_{2}\left\|f_{2}\right\|_{\infty}\left\|f_{3}\right\|_{\infty} .
$$

On the other hand, applying the definition of $\mathcal{A}$ to the off-spring curve and the fact that the measure of $\left\{\left(t_{2}, t_{3}\right):\left(t_{1}, t_{2}, t_{3}\right) \in S_{k}\right\}$ is $O\left(2^{-2 k / 3}\right)$ leads to

$$
\left\|\mathcal{M}_{k}\left(f_{1}, f_{2}, f_{3}\right)\right\|_{L^{7, \infty}\left(B_{R}\right)} \leq C \mathcal{A}_{\delta}(b, 3 R) 2^{-2 k / 3}\left\|f_{1}\right\|_{7}\left\|f_{2}\right\|_{\infty}\left\|f_{3}\right\|_{\infty} .
$$

From here on we argue as in the proof of Theorem 1.2. Applying Bourgain's interpolation lemma we get

$$
\left\|\mathcal{M}\left(f_{1}, f_{2}, f_{3}\right)\right\|_{L^{7 / 3, \infty}\left(B_{R}\right)} \leq C \delta^{-2 / 5} \mathcal{A}_{\delta}(b, 3 R)^{1 / 5}\left\|f_{1}\right\|_{7 / 3,1}\left\|f_{2}\right\|_{\infty}\left\|f_{3}\right\|_{\infty}
$$

and applying the multilinear interpolation arguments to the symmetric $n$ linear expression $\prod_{i=1}^{n} \mathcal{E} f_{i}$, for $n>7$ (e.g. $n=21$ as in $\S 3$ ) yields

$$
\left\|\prod_{i=1}^{n} \mathcal{E} f_{i}\right\|_{L^{7 / n, \infty}\left(B_{R}\right)} \leq C \delta^{-2 n / 15} \mathcal{A}_{\delta}(b, 3 R)^{n / 15} \prod_{i=1}^{n}\left\|f_{i}\right\|_{L^{7, r_{i}(I)}}
$$

where $\sum_{i=1}^{n} r_{i}^{-1}=n / 7$. We may set $f_{i}=f, r_{i}=7$ and obtain

$$
\mathcal{A}_{\delta}(b, R) \leq C \delta^{-2 / 15} \mathcal{A}_{\delta}(b, 3 R)^{1 / 15}
$$

and since from definition (7.5) it follows that $\mathcal{A}_{\delta}(b, 3 R) \lesssim \mathcal{A}_{\delta}(b, R)$ we obtain $\mathcal{A}_{\delta}(b, R) \lesssim \delta^{-1 / 7}$ which is the assertion.

Conclusion of the proof of Theorem 1.4. We first consider part (i). By symmetry we may assume $\alpha \leq 2$. The cases $\alpha=0$ and $\alpha=1$ are trivial since then $w_{\gamma} \equiv 0$, and the case $\alpha=2$ has been already handled in $\S 3$. Thus suppose $\alpha=5-\beta<2$, and $\alpha \neq\{0,1\}$. Then by the discussion in the beginning of this section the affine arclength measure is $c_{\alpha}^{1 / 6}(5-2 \alpha)^{1 / 6} d t$ with $c_{\alpha}=|\alpha(5-\alpha)(\alpha-1)(4-\alpha)|$. Moreover $\Delta(s, t)=$ 
$c_{\alpha}\left|(3-\alpha) s^{\alpha-2} t^{2-\alpha}+(2-\alpha) s^{3-\alpha} t^{\alpha-3}\right|$ and $c_{\alpha}^{-1} \Delta(s, t)$ has its minimum $(5-2 \alpha)$ at $(1,1)$. From this part (i) of the theorem follows easily. Part (ii) follows from part (i) by the change of variable $u=t^{\alpha}$, and interchanging the first and second components of $\gamma$.

\section{Appendix A.}

\section{VANDERmonde operators: Proof of the Drury-Marshall Bound}

For the sake of self-containedness we give the full proof of Proposition 2.4, due to Drury and Marshall. This is done by first checking (i) for $d=2$ and $d=3$, and then by arguing by induction, applying a special case of (ii) in $d-2$ dimensions to prove (i) and (ii) in $d$ dimensions.

We note that by a homogeneity argument it suffices to prove that the set $\Omega_{d}(1)$ has finite measure in $\mathbb{R}^{d-1}$. It is obvious that the measure of $\Omega_{2}(1)$ is equal to 1 . If $d=3$ then $v_{3}(h)=V_{3}\left(\kappa\left(h_{1}, h_{2}\right)\right)=h_{1} h_{2}\left(h_{1}+h_{2}\right)$ and the set $\left\{h: v_{3}(h) \leq 1\right\}$ is contained in the union of two sets $E_{1} \cup E_{2}$ where $E_{1}=\left\{h \in(0, \infty)^{2}: h_{1} h_{2}^{2} \leq 1, h_{1} \leq h_{2}\right\}$ and $E_{2}=\left\{\left(h_{1}, h_{2}\right):\left(h_{2}, h_{1}\right) \in E_{1}\right\}$. Both sets have area equal to $\int_{0}^{\infty} \min \left\{s, s^{-2}\right\} d s=3 / 2$.

Now we assume that (ii) has been established in all dimensions $\leq d-1$, and we shall prove that $\Omega_{d}(1)$ has finite measure in $\left(\mathbb{R}_{+}\right)^{d-1}$, and that $(2.10)$ holds in $d$ dimensions.

We now set $r \equiv r(h)=\kappa_{d}(h)=h_{1}+\cdots+h_{d-1}, t \equiv t(h)=h_{1} / \kappa_{d}(h)$ and $\tau_{i}(h)=h_{i+1} / \kappa_{d}(h), i=1, \ldots, d-3$. We use the change of variable $h \mapsto$ $\left(t, \tau_{1}, \ldots, \tau_{d-3}, r\right)$ and observe the determinant of its derivative is $r(h)^{-d+2}$.

Set $\tilde{\kappa}(\tau)=\left(\tilde{\kappa}_{1}, \ldots, \tilde{\kappa}_{d-3}\right)$, with $\tilde{\kappa}_{1}(\tau)=0$ and $\tilde{\kappa}_{i}(\tau)=\sum_{k=1}^{i-1} \tau_{k}$, for $2 \leq i \leq d-2$. Then we can write

$$
\begin{aligned}
& v_{d}(h)=\left(\prod_{j=2}^{d} \kappa_{j}(h)\right)\left(\prod_{2 \leq i<j \leq d-1} \kappa_{j}(h)-\kappa_{i}(h)\right)\left(\prod_{k=2}^{d-1}\left(\kappa_{d}(h)-\kappa_{i}(h)\right)\right) \\
& =\kappa_{d}(h)^{\frac{d(d-1)}{2}}\left(\prod_{j=2}^{d} \frac{\kappa_{j}(h)}{\kappa_{d}(h)}\left(1-\frac{\kappa_{j}(h)}{\kappa_{d}(h)}\right)\right)\left(\prod_{2 \leq i<j \leq d-1} \frac{\kappa_{j}(h)-\kappa_{i}(h)}{\kappa_{d}(h)}\right) \\
& =r(h)^{\frac{d(d-1)}{2}} \prod_{j=1}^{d-2}\left[\left(t+\tilde{\kappa}_{j}(\tau)\right)\left(1-t-\tilde{\kappa}_{j}(\tau)\right)\right]\left(\prod_{1 \leq i<j \leq d-2}\left(\tilde{\kappa}_{j}(\tau)-\tilde{\kappa}_{i}(\tau)\right)\right) .
\end{aligned}
$$

Thus, if $U(s):=\left(\prod_{i=1}^{d-2}\left[s_{i}\left(1-s_{i}\right)\right]\right) V_{d-2}(s)$, defined on $\Sigma_{d-2}:=\left\{s \in \mathbb{R}^{d-2}\right.$ : $\left.0 \leq s_{1} \leq \ldots \leq s_{d-2} \leq 1\right\}$, then

$$
\begin{aligned}
\left|\Omega_{d}(1)\right| & \leq \int_{\Sigma_{d-2}} \int_{0}^{U(s)^{-\frac{2}{d(d-1)}}} r^{d-2} d r d s \leq \int_{\Sigma_{d-2}} U(s)^{-2 / d} d s \\
& =\int_{\left(\mathbb{R}_{+}\right)^{d-3}} \int_{0}^{\infty} \prod_{i=1}^{d-2}\left|g\left(t+\tilde{\kappa}_{i}(\tau)\right)\right|^{A} d t\left[v_{d-2}(\tau)\right]^{1-A} d \tau
\end{aligned}
$$


here $v_{d-2}(\tau):=V_{d-2}(\tilde{\kappa}(\tau)), A=(d+2) / d, g(s)=s(1-s)^{-2 /(d+2)} \chi_{[0,1]}(s)$. Thus the last expression is the $A^{\text {th }}$ power of the $L_{v}^{A}\left(L^{A}\right)$ norm of the Vandermonde operator in $d-2$ dimension, applied to the functions $f_{i}=g$, $i=1, \ldots, d-2$. The value $A=(d+2) / d<d /(d-2)$ is permissible for the application of part (ii) in $(d-2)$ dimensions. Now with $\sigma=2(d-(d-2) A)^{-1}=$ $d / 2$ we need to verify that $g \in L^{p, 1}$ with $(d-2) / p=A^{-1}(1+(d-3) / \sigma)$ (which corresponds to the point in the center of the $(d-3)$-dimensional simplex $\Sigma(A, A))$. Note that $p=(d+2) / 3$, and as $g$ belongs to $L^{r}[0,1]$ for all $r<(d+2) / 2$ it belongs surely to $L^{p, 1}$. Thus part (i) is verified in $d$ dimensions.

We now turn to the proof of (2.10) in $d$ dimensions. First notice that the allowable $p_{i}$ 's are given by the equation $\sum_{i=1}^{d} p_{i}^{-1}=(d-1)(\sigma A)^{-1}+B^{-1}$ and that $\left(p_{1}^{-1}, \ldots, p_{d}^{-1}\right)$ belongs to $\Sigma(A, B)$ if and only if

$$
\frac{1}{p_{i}}=\frac{1}{\sigma A}+\left(\frac{1}{B}-\frac{1}{\sigma A}\right) \frac{1}{r_{i}}
$$

where $r_{i} \in[1, \infty]$ with $\sum_{i=1}^{d} r_{i}^{-1}=1$.

It suffices to prove the estimate (2.10) for $f_{i}$ which is pointwise dominated by characteristic functions of measurable sets $E_{i}, i=1, \ldots, d$, and by monotonicity properties of the operator we may assume that $f_{i}=\chi_{E_{i}}$. Thus we need to prove

$$
\left\|\mathfrak{V}\left(\chi_{E_{1}}, \ldots, \chi_{E_{d}}\right)\right\|_{L_{v}^{A}\left(L^{B}\right)} \leq C \prod_{i=1}^{d}\left|E_{i}\right|^{1 / p_{i}} .
$$

We use a duality argument for the $h$ integral in (2.9). By part (i) the function $|v|^{1-A}$ belongs to $L^{\sigma^{\prime}, \infty}\left(\mathbb{R}^{d-1}\right)$ for $\sigma^{\prime}=2 d^{-1}(A-1)^{-1}$. Note that because of our assumption on $A$ we have $\sigma^{\prime} \in(1, \infty)$; moreover $\sigma^{\prime}$ is the conjugate exponent to $\sigma=2 /(2+d-d A)$. Define

$$
\Phi(h)=\int \prod_{i=1}^{d}\left|\chi_{E_{i}}\left(t+\kappa_{i}(h)\right)\right| d t,
$$

as a function defined on $(0, \infty)^{d-1}$. As $\chi_{E_{i}}$ assumes only the values 0 and 1 it suffices to show that $\left\|\Phi^{A / B}\right\|_{L^{\sigma, 1}} \lesssim \prod_{i=1}^{d}\left|E_{i}\right|^{A / p_{i}}$ which follows from

$$
\|\Phi\|_{L^{A \sigma / B, A / B}} \leq C \prod_{i=1}^{d}\left|E_{i}\right|^{\frac{B}{p_{i}}} .
$$

We now use the familiar inequality

$$
\|G\|_{L^{P, s}} \leq C(P, s)\|G\|_{1}^{1 / P}\|G\|_{\infty}^{1-1 / P}
$$

which holds for all $P \in(1, \infty)$ and all $s \in(0, \infty)$; and we apply this for $G \equiv \Phi$, and $P=A \sigma / B>1, s=A / B \in(0,1]$. It is easy to see that 
$\|\Phi\|_{1} \leq \prod_{i=1}^{d}\left|E_{i}\right|$ and, by Hölder's inequality, $\|\Phi\|_{\infty} \leq \prod_{i=1}^{d}\left|E_{i}\right|^{1 / r_{i}}$ for all $r_{1}, \ldots, r_{d} \in[1, \infty]$ satisfying $\sum_{i=1}^{d} r_{i}^{-1}=1$. Now by (A.3),

$$
\|\Phi\|_{L^{A \sigma / B, A / B}} \leq c(A, B) \prod_{i=1}^{d}\left|E_{i}\right|^{\frac{B}{A \sigma}+\left(1-\frac{B}{A \sigma}\right) \frac{1}{r_{i}}}
$$

where $r_{i} \in[1, \infty]$ with $\sum_{i=1}^{d} r_{i}^{-1}=1$. By the above description of the simplex $\Sigma(A, B)$ this inequality yields (A.2) and thus the assertion.

\section{REFERENCES}

[1] G.I. Arkhipov, V.N. Chubarikov, and A.A. Karatsuba, Exponent of convergence of the singular integral in the Tarry problem. (Russian) Dokl. Akad. Nauk SSSR, 248 (1979), no. 2, $268-272$.

[2] Trigonometric sums in number theory and analysis. Translated from the 1987 Russian original. de Gruyter Expositions in Mathematics, 39, Berlin, 2004.

[3] J.-G. Bak, S. Lee, Estimates for an oscillatory integral operator related to restriction to space curves, Proc. Amer. Math. Soc. 132 (2004), 1393-1401.

[4] J.-G. Bak, D. Oberlin, A note on Fourier restriction for curves in $\mathbb{R}^{3}$, Proceedings of the AMS Conference on Harmonic Analysis, Mt. Holyoke College (June 2001), Contemp. Math., Vol. 320, Amer. Math. Soc., Providence, RI, 2003.

[5] J.-G. Bak, D. Oberlin, A. Seeger, Restriction of Fourier transforms to curves, II: Some classes with vanishing torsion, J. Austr. Math. Soc., to appear.

[6] W. Beckner, A. Carbery, S. Semmes, F. Soria, A note on restriction of the Fourier transform to spheres, Bull. London Math. Soc. 21 (1989), 394-398.

[7] J. Bergh, J. Löfström, Interpolation spaces. An introduction. Grundlehren der Mathematischen Wissenschaften, No. 223. Springer-Verlag, Berlin-New York, 1976.

[8] J. Bourgain, Estimations de certaines fonctions maximales, C. R. Acad. Sci. Paris Sér. I Math. 301 (1985), no. 10, 499-502.

[9] L. Brandolini, G. Gigante, A. Greenleaf, A. Iosevich, A. Seeger, G. Travaglini, Average decay estimates for Fourier transforms of measures supported on curves, J. Geom. Anal., 17 (1) (2007), 15-40.

[10] A. Carbery, Differentiation in lacunary directions and an extension of the Marcinkiewicz multiplier theorem, Annales de l'institut Fourier 38, no. 1 (1988), p. 157-168.

[11] A. Carbery, A. Seeger, S. Wainger and J. Wright, Classes of singular integral operators along variable lines, J. Geom. Anal. 9 (1999), no. 4, 583-605.

[12] L. Carleson, P. Sjölin, Oscillatory integrals and a multiplier problem for the disc, Studia Math. 44 (1972), 287-299.

[13] M. Christ, On the restriction of the Fourier transform to curves: endpoint results and the degenerate case, Trans. Amer. Math. Soc. 287 (1985), 223-238.

[14] S.W. Drury, Restriction of Fourier transforms to curves, Ann. Inst. Fourier, 35 (1985), 117-123.

[15] Degenerate curves and harmonic analysis, Math. Proc. Cambridge Philos. Soc. 108 (1990), 89-96.

[16] S.W. Drury, B. Marshall, Fourier restriction theorems for curves with affine and Euclidean arclengths, Math. Proc. Cambridge Philos. Soc. 97 (1985), 111-125.

[17] _ Fourier restriction theorems for degenerate curves, Math. Proc. Cambridge Philos. Soc. 101 (1987), 541-553.

[18] C. Fefferman, Inequalities for strongly singular convolution operators, Acta Math. 124 (1970), 9-36.

[19] L. Hörmander, Oscillatory integrals and multipliers on $F L^{p}$, Ark. Mat. 11 (1973), $1-11$. 
[20] R. Hunt, On $L(p, q)$ spaces, Enseignement Math. (2), 12 (1966), 249-276.

[21] S. Janson, On interpolation of multilinear operators. Function spaces and applications (Lund, 1986), 290-302, Lecture Notes in Math., 1302, Springer, Berlin, 1988.

[22] N. J. Kalton, Linear operators on $L^{p}$ for $0<p<1$, Trans. Amer. Math. Soc. 259 (1980), 319-355.

[23] U. Keich, On $L^{p}$ bounds for Kakeya maximal functions and the Minkowski dimension in $\mathbb{R}^{2}$, Bull. London Math. Soc. 31 (1999), no. 2, 213-221.

[24] G. Mockenhaupt, Bounds in Lebesgue spaces of oscillatory integral operators. Habilitationsschrift, Universität Siegen (1996).

[25] A. Nagel, E.M. Stein, S. Wainger, Differentiation in lacunary directions, Proc. Nat. Acad. Sci. U.S.A., 75 (1978), no. 3, 1060-1062.

[26] D. M. Oberlin, Fourier restriction estimates for affine arclength measures in the plane, Proc. Amer. Math. Soc. 129 (2001), 3303-3305.

[27] G. Pólya, G. Szegö, Aufgaben und Lehrsätze aus der Analysis II, 4th ed. Springer 1970. English Translation: Die Grundlehren der mathematischen Wissenschaften, Band 193. Springer-Verlag, New York-Berlin, 1972.

[28] E. Prestini, Restriction theorems for the Fourier transform to some manifolds in $R^{n}$, Proc. Sympos. Pure Math. 35 (1979), 101-109.

[29] Y. Sagher, On analytic families of operators, Israel J. Math. 7 (1969), 350-356.

[30] P. Sjölin, Fourier multipliers and estimates of the Fourier transform of measures carried by smooth curves in $R^{2}$, Studia Math., 51 (1974), 169-182.

[31] C.D. Sogge, A sharp restriction theorem for degenerate curves in $R^{2}$, Amer. J. Math. 109 (1987), no. 2, 223-228.

[32] E.M. Stein, M. Taibleson, G. Weiss, Weak type estimates for maximal operators on certain $H^{p}$ classes. Proceedings of the Seminar on Harmonic Analysis (Pisa, 1980). Rend. Circ. Mat. Palermo (2), 1981, suppl. 1, 81-97.

[33] E.M. Stein, G. Weiss, Introduction to Fourier analysis on Euclidean spaces, Princeton Mathematical Series, No. 32. Princeton University Press, Princeton, N.J., 1971.

[34] E.M. Stein, N.J. Weiss, On the convergence of Poisson integrals, Trans. Amer. Math. Soc. 140 (1969), 35-54.

[35] T. Tao, Sharpness of the Carleson-Sjölin theorem, expository note, 2001.

[36] P.A. Tomas, A note on restriction, Indiana Univ. Math. J. 29 (1980), no. 2, 287-292.

[37] A. Zygmund, On Fourier coefficients and transforms of functions of two variables. Studia Math. 50 (1974), 189-201.

J. Bak, Department of Mathematics and the Pohang Mathematics Institute, Pohang University of Science and Technology, Pohang 790-784, Korea

E-mail address: bak@postech.ac.kr

D. M. Oberlin, Department of Mathematics, Florida State University, TalLAHASSEE, FL 32306

E-mail address: oberlin@math.fsu.edu

A. Seeger, Department of Mathematics, University of Wisconsin-Madison, MADISON, WI 53706, USA

E-mail address: seeger@math.wisc.edu 